CrossMark \&lick for updates

Cite this: Soft Matter, 2017, 13,304

Received 12th September 2016, Accepted 21st November 2016

DOI: $10.1039 / c 6 s m 02078 j$

www.rsc.org/softmatter

\section{Surface tension-driven self-alignment $\dagger$}

\author{
Massimo Mastrangeli, ${ }^{\star a}$ Quan Zhou, $^{\mathrm{b}}$ Veikko Sariolac ${ }^{\mathrm{c}}$ and Pierre Lambert ${ }^{\mathrm{d}}$
}

Surface tension-driven self-alignment is a passive and highly-accurate positioning mechanism that can significantly simplify and enhance the construction of advanced microsystems. After years of research, demonstrations and developments, the surface engineering and manufacturing technology enabling capillary self-alignment has achieved a degree of maturity conducive to a successful transfer to industrial practice. In view of this transition, a broad and accessible review of the physics, material science and applications of capillary self-alignment is presented. Statics and dynamics of the self-aligning action of deformed liquid bridges are explained through simple models and experiments, and all fundamental aspects of surface patterning and conditioning, of choice, deposition and confinement of liquids, and of component feeding and interconnection to substrates are illustrated through relevant applications in micro- and nanotechnology. A final outline addresses remaining challenges and additional extensions envisioned to further spread the use and fully exploit the potential of the technique.
${ }^{a}$ Physical Intelligence Department, Max Planck Institute for Intelligent Systems, Max Planck ETH Center for Learning Systems, Heisenbergstr. 3, 70569 Stuttgart, Germany.E-mail: mastrangeli@is.mpg.de

${ }^{b}$ Department of Electrical Engineering and Automation, School of Electrical Engineering, Aalto University, Otaniementie 17, 02150 Espoo, Finland

${ }^{c}$ Department of Automation Science and Engineering, Tampere University of Technology, Korkeakoulunkatu 3, 33720 Tampere, Finland

${ }^{d}$ Department of Bio, Electro And Mechanical Systems, École Polytechnique de Bruxelles, Université Libre de Bruxelles, CP 165/56. Avenue F.D. Roosevelt 50, 1050 Brussels, Belgium

$\dagger$ Electronic supplementary information (ESI) available. See DOI: 10.1039/c6sm02078j

\section{Introduction}

Understanding the role that surface tension plays in the multiform interactions between liquid phases and solid surfaces drives a significant part of current research in fluidics, ${ }^{1}$ mechanics, ${ }^{2}$ soft matter ${ }^{3}$ and interfacial engineering. ${ }^{4}$ Practical implications of new insights gained from basic research can rapidly lead to applications in wetting and surface conditioning, ${ }^{5}$ precision engineering, ${ }^{6}$ colloidal assembly, ${ }^{7}$ microfluidics ${ }^{8}$ and micro/nanofabrication..$^{9}$ In particular, the accurate and repeatable manipulation of micro- and nanoscopic objects represents a

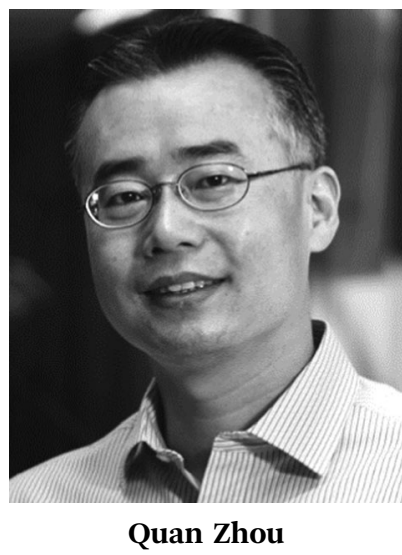

Quan Zhou received the MSc degree in Control Engineering and Dr Tech. degree in Automation Technology, both from Tampere University of Technology, Tampere, Finland. He is currently Associate Professor in Automatic Control at the Department of Electrical Engineering and Automation, School of Electrical Engineering, Aalto University, Finland, heading the Micro- and Nanorobotics research group. His main research interests are micro- and nanorobotic manipulation and automation methods and their applications in both industry and biomedical applications. Prof. Zhou was also the coordinator of EU FP7 project FAB2ASM, the first PPP project of the European Economic Recovery Plan. 
technological field of far-reaching industrial, biomedical and commercial relevance that is vastly benefiting from such research. ${ }^{10}$ In this context, for the past two decades fluidmediated manipulation has demonstrated to be capable of simplifying and enhancing the construction of heterogeneous micro- and nanosystems.

Precise positioning is of foremost importance when assembling together sets of modules or devices that are functionally correlated. Recent advances in miniaturization, especially in the pitch and diameter of interconnections, and in the integration of diverse complementary functions often make the realization of novel microelectronic, optoelectronic and nanoscale systems possible only through extremely accurate registration among the modules. Robotic assembly ${ }^{10}$ and stochastic self-assembly ${ }^{9}$ represent the opposite extremes of a range of approaches to precision assembly. ${ }^{11}$ Robotic pick-and-place is the current standard in precision industrial assembly. It achieves impressive performance (on the order of tens of thousands of assembled components per hour in the case of flip-chip assembly machines ${ }^{12}$ ) through the supervised dexterity of robotic effectors. The throughput of robotic pick-and-place is however limited by its seriality, while its accuracy degrades when handling components of sub-millimetric size. ${ }^{13}$ Self-assembly is an alternative, bio-inspired and intrinsically parallel technique for high-throughput manufacturing, ${ }^{12}$ particularly for handling very small, ultrathin and flexible components. Surface tension-driven (alias capillary) self-alignment (Fig. 1) lies somewhat in the middle of the assembly spectrum, as it merges deterministic aspects of component handling and feeding with passive, unsupervised and very accurate component registration. When compared to stochastic fluidic self-assembly, the key advantage of capillary self-alignment is that its steps can more easily be individuated, parameterized and streamlined into industrial process flows.

The development of capillary self-alignment progressed across three subsequent phases. In the first phase, the surface tension of molten solder-based interconnections was used to self-align and electromechanically fix surface-mount components to electronic substrates - a process originally introduced in the ' $60 \mathrm{~s}^{14}$ which came to be generally associated with flip-chip assembly. Through continuous evolution, including its adaptation to threedimensional capillary deployment of microelectromechanical systems (MEMS) $)^{15}$ and capillary self-folding of micro- and nanoscopic voxels,${ }^{16}$ presently molten solder-based self-alignment is amply used in advanced microelectronic integration processes involving solder microbumps, through-silicon vertical interconnect accesses (VIAs) and three-dimensional chip stacking to meet ultimate performance and input/output requirements. The second phase, started in the late ' 90 s, saw the development of a multitude of fluidic self-assembly approaches. Among them, capillarity-driven component-to-substrate stochastic selfassembly emerged as the most convenient for industrial adoption. In the third phase and during the last decade, the fluidic self-assembly process was adapted to work in an air environment. This evolution is akin to a return to the origin and enabled the combination with robotic component feeding into what was called hybrid microhandling. ${ }^{17}$ Such latest embodiment indeed consists of an extension of the IBM's original controlled-collapse chip connection (C4) integration process ${ }^{14}$ whereby an additional liquid bridge wraps and complements the function of the solder bumps. ${ }^{18}$

The outlined stages of development tackled advances in technology and materials science, among which in particular surface conditioning and patterning, wetting control, new interconnection materials, ultrathin and flexible components. While most of past and current research concerned inorganic components, capillary self-alignment may hold potential also for organic assemblies. The understanding of the impact of process parameters - such as surface tension, viscosity and contact angle of liquids, geometry of components and receptors, relative misalignment - also gradually improved thanks to the development of analytical and numerical models. While the theoretical picture of capillary self-alignment, especially regarding its dynamics, is not fully clear yet, the available frameworks have contributed to increase assembly throughput and yield through better process design.

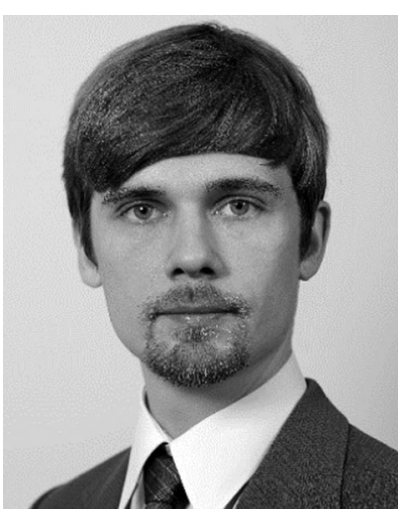

Veikko Sariola
Veikko Sariola is Assistant Professor in Bio-MEMS at the Tampere University of Technology, in Finland. In 2012, he obtained his Dr Tech. degree in Electrical Engineering from Aalto University, Finland. From 2013 to 2015, he was a post-doctoral researcher at Carnegie Mellon University, and in 2016, he was appointed as a Research Fellow of the Academy of Finland. His current research interests include micromanipulation, wetting, bioinspired materials, and robotics.

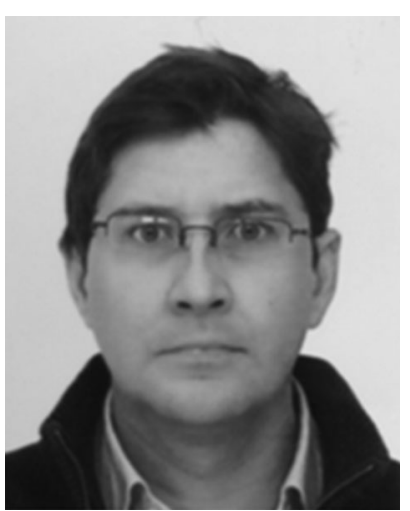

Pierre Lambert
Pierre Lambert is Associate Professor in Mechanical Engineering at Université libre de Bruxelles. His researches are in the field of microengineering (" microtechniques »), with a special focus on flexible mechanisms (soft robotics) and surface tension effects. He published about 40 journal papers in these fields, and 2 patents. He is the coordinator of the Belgian thematic network on "Microfluidics and Micromanipulation: Multiscale Applications of Surface Tension (IAP 7/38 MicroMAST)", funded by the Belgian science office (BELSPO). 
(a)

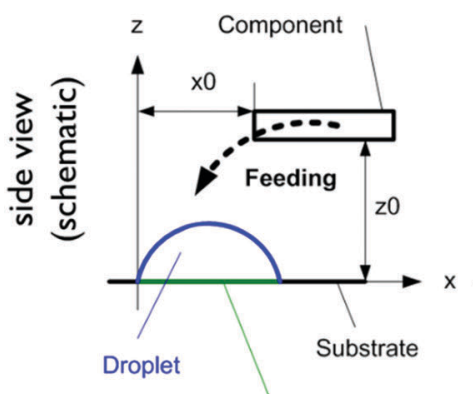

Receptor

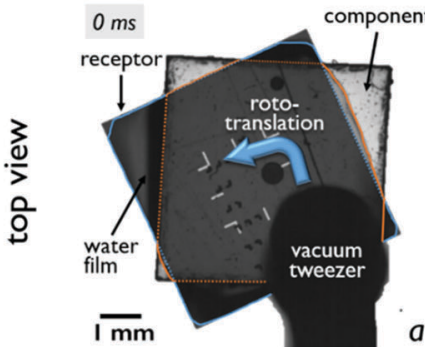

(b)

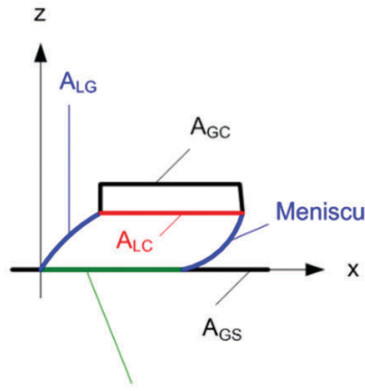

$A_{L R}$

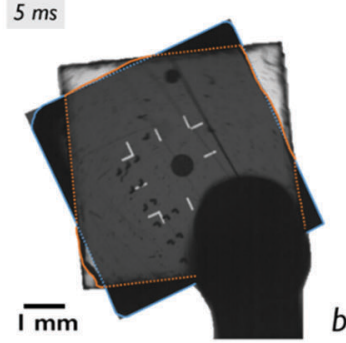

(c)

(d)

Fig. 1 Capillary self-alignment. Top row: Sketch of the process. (a) A component is brought in the vicinity of a droplet confined on a receptor site at offsets $x_{0}, y_{0}$ (not shown) and $z_{0}$ with respect to its final position; (b) description of the process based on the minimization of the surface energy (see Section 2.3); (c) dual description based on a capillary force vector acting on the component (Section 2.4); (d) end of the alignment process, with possible residual misalignment. Bottom row: Sequential snapshots of the capillary self-alignment of a square transparent plastic component onto a shapematching receptor coated with a thin water film. The autonomous roto-translational motion of the component starts upon its release from the tweezer only after the triple contact line reaches at least one of the solid edges of the bottom surface of the component. Final placement of the component on the receptor and alignment of the edges is achieved after a few oscillation cycles (not shown) due to underdamped dynamics. (Adapted with permission from ref. 20 (C) 2014, American Chemical Society.)

This review is tailored to guide the readers through the practice of capillary self-alignment. The underlying physics and the material requirements of the process are described together with the state-of-the-art in its performance and achievements. The paper is intended as a tutorial to help transpose prior experimental demonstrations into industrial practice. Recent works $^{19}$ and patents support the claim that capillary selfalignment's current state of development is compatible with this forthcoming step.

The paper is structured as follows. Definitions and fundamental concepts concerning wetting and triple contact lines are first summarized to introduce an overview and the governing physics of surface tension-driven self-alignment (Section 2). The design of constrained liquid bridges is then addressed, highlighting the importance of surface properties in the methods of liquid confinement (Section 3) and the role of shape matching between component and receptor (Section 4). Section 5 provides an overview of the dynamics of capillary selfalignment, as reflected in models and experimental data. Process integration is discussed in the following Section 6, concerning the choice of liquids, techniques for controlled liquid deposition and feeding of components to the receptors, and available options to electrically besides mechanically bind components to substrates upon completion of capillary selfalignment. Relevant applications of surface tension-driven selfalignment reported in recent literature are also exemplified, and a survey of related patents is provided in Section 7 .
Open challenges and future perspectives are outlined in the concluding Section 8.

\section{Fundamentals of surface-tension- driven self-alignment}

\subsection{Definitions and terminology}

Surface-tension-driven self-alignment (also known as capillary self-alignment) is a fluid-based technology used to accurately align components to patterns or features of a substrate. When a confined liquid drop is interposed between a suitably patterned region of a substrate and a freely moving component, the droplet forms a liquid meniscus whose constrained capillary action tends to align the component to the pattern. The attribute "self" refers to the apparent autonomy of the transient component motion. A sketch of the process and sequential snapshots from a capillary self-alignment experiment are shown in Fig. 1.

The object to be assembled - typically a chip, die, device or functional module in electronics applications - is here called component (C). The well-defined area $A_{\mathrm{LR}}$, on a substrate or on another component, to which the component is required to self-align represents the binding, assembly or target site, hereby referred to as receptor (R). The finite liquid phase $\mathrm{L}$, bounded by the gas phase $\mathrm{G}$ and interposed between the component and the receptor to form a liquid bridge or meniscus, ${ }^{4}$ additionally prevents dry friction between the sliding surfaces 
of component and receptor and hence is sometimes called lubricant. The height $h$ of the liquid bridge defines the gap.

Most of the emphasis in capillary self-alignment is reserved to in-plane motion, i.e. the biaxial $(x, y)$ and rotational $\left(\theta_{z}\right)$ motion of the component relative to the receptor. This represents a simplified account, as the alignment process inevitably involves perturbations and motion of the component along all six degrees of freedom of the fluid joint, ${ }^{2,4}$ whereby vertical motion $(z)$ and out-of-plane rotations $\left(\theta_{x}\right.$ and $\left.\theta_{y}\right)$ are also included. The emphasis on lateral registration derives from technological applications, such as precision assembly tasks and micro/ nanoelectronic integration, and has in turn largely biased the study and modelling of the process.

In terms of component placement, the bias or offset refers to the initial distance (i.e. prior to the inception of the selfaligning motion) of the component to its final expected position - typically the one where the edges of the component are perfectly aligned to those of the receptor. The offset vector identifies the component position at the time the component is released, in case of robotic feeding, or when the component is first wet by the droplet, in case of stochastic feeding (Fig. 1a). Misalignment is conversely a measure of registration error, and refers to the eventual residual distance between actual and expected position of the component after completion of the self-alignment process (Fig. 1d, top row). Misalignment may arise from wetting defects (Section 3.2) and shape mismatch (Section 4.2). Null misalignment identifies correct final component positioning. Both offset and misalignment can be measured either absolutely or relatively to the dimensions of the component or receptor. Offset and misalignment are sometimes also measured by the distance between corresponding edges of component and receptor, respectively at the beginning and at the end of the process.
The (alignment) yield of capillary self-alignment indicates the fraction of components that end up being correctly positioned after process completion on a selected number of receptors (i.e., 9 correctly aligned components out of 10 delivered onto 10 receptors corresponds to a yield of 90\%). Successful alignment is usually defined by compliance with targeted misalignment margins. The margins are in turn specifically determined by the applications at hand. Beside alignment yield, functional yield is relevant in the assembly of systems whereby the mechanical connection needs to provide also an electrical path between receptor and component. Functional assembly yield is altogether a more restrictive success metric, since typically only a subset of the correctly aligned components can also sustain electrical functionality. For instance, in the packaging of advanced integrated circuits onto back-end-of-line (BEOL)-processed substrates, sub-micrometric post-alignment registration accuracy is being targeted to enforce working electrical interconnections through solder microbumps. ${ }^{18}$ Finally, the overall efficiency of the process is measured by its throughput, which quantifies yield per unit of time. Sections 3, 4 and 6 and Mastrangeli et al. ${ }^{21}$ provide an overview of major failure modes in capillary selfalignment and fluidic self-assembly.

\subsection{Wetting and contact lines}

A drop (Fig. 2a) is a finite liquid phase delimited by an interface which is the seat of the surface tension phenomenon. ${ }^{22}$ The excess surface energy of the interface is its surface tension $\gamma$. When a liquid drop is in contact with a solid substrate, two additional interfaces besides the liquid-gas (LG) are identified: the liquid-solid (LS) interface and the solid-gas (SG) interface elsewhere (Fig. 2b). The intersection between the surfaces tangent respectively to the solid-liquid and the gas-liquid interfaces is called the (triple) contact line. The angle defined a

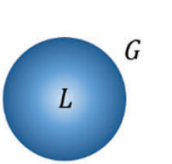

$b$
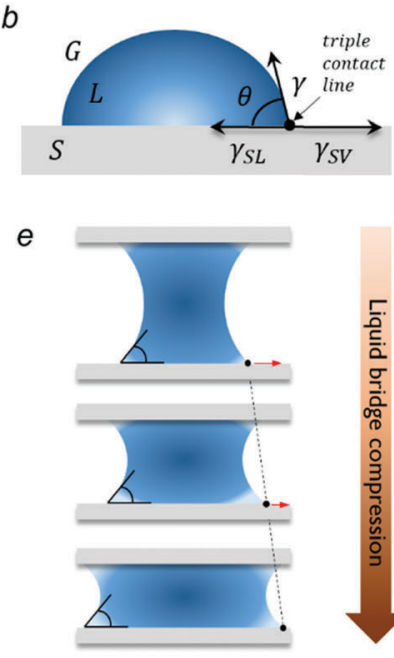

Constant contact angle $c$

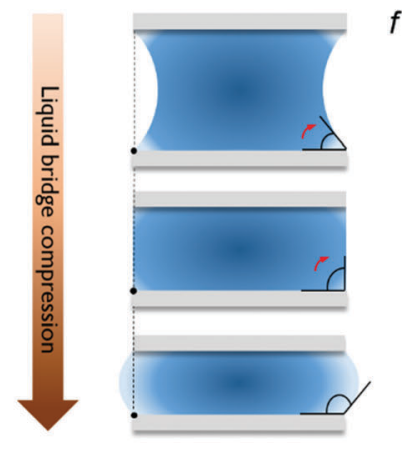

Pinned contact line

Fig. 2 (a) A suspended liquid drop (without support); (b) a liquid drop wetting a rigid substrate without solid edge, defining a triple contact line and a static contact angle; (c) a liquid drop wetting a rigid substrate with a solid edge; (d) a liquid bridge, i.e. a liquid drop bridging two separated solid surfaces. (e) Moving contact line with constant prescribed (advancing) contact angle, versus (f) pinning of the contact line at a fixed position imposed by a solid edge. The compression of the liquid bridges is in both cases consequent to the reduction of the gap between the parallel bounding surfaces. 
by both tangent planes and spanning the liquid phase is called the contact angle $\theta$. The contact angle governs the capillary forces exerted by the liquid on the solid (see Section 2.4). Usually, the tangent to the solid-gas interface coincides with the tangent to the solid-liquid interface. There exist however situations where this is not the case, for instance when the contact line wets a sharp solid edge (see Fig. 2c and Section 3.1). When the droplet makes contact with separated solid surfaces, it is usually referred to as a meniscus or liquid bridge (Fig. 2d).,23

The contact line is in principle free to move across a substrate with chemically homogeneous and topographically smooth surface (Fig. 2e). At equilibrium, in proximity of the contact line the meniscus will eventually assume on a sufficiently rigid substrate a shape set by the equilibrium contact angle, which, although ill-defined, is typically described by the Young-Dupré equation $\cos \theta=\left(\gamma_{\mathrm{SG}}-\gamma_{\mathrm{LS}}\right) / \gamma$. The equation captures the balance of the tension components parallel to the substrate. A low $\theta$ signals high chemical affinity of the liquid to the substrate, and perfect wetting is achieved for $\theta \rightarrow 0$. Over rough and/or chemically heterogeneous surfaces, a range of apparent contact angles can instead be observed at a given contact line position (Fig. 2f). Contact line movement will then only occur when the apparent contact angle is either larger than an upper threshold value, called the advancing contact angle $\theta_{\mathrm{a}}$, or smaller than a lower threshold value, called the receding contact angle $\theta_{\mathrm{r}}$. The angular sector $\left[\theta_{\mathrm{r}} \theta_{\mathrm{a}}\right]$ between both threshold values quantifies the contact angle hysteresis. When the contact angle lies within such interval, the contact line is said to be pinned. Contact line pinning, whose microscopic origin is attributed either to localized defects or adhesion hysteresis, ${ }^{24}$ can be further reinforced by the presence of sharp edges on the substrates (Fig. 2c). The increases in contact angle hysteresis induced in this case by slope discontinuity can be exploited for topographical liquid confinement, as described in Section 3.1.

For a geometrical description of meniscus shape, the knowledge of the contact line position is needed when the contact line is pinned (Dirichlet boundary condition), while the knowledge of the contact angle must be used when the contact line is moving or its position is unconstrained (Neumann boundary condition). ${ }^{23}$ The dynamic contact angle is also dependent on the velocity of the contact line. ${ }^{25}$ The contact line can switch from being pinned to mobile during a single experiment according to changing conditions. For instance, in the early instants after deposition on a homogeneous solid surface, a droplet relaxes and the liquid spreads over the substrate until its motion stops upon reaching an equilibrium configuration or a boundary. Similarly, when a component is set on top of a confined liquid droplet, either by release or by pre-contact, ${ }^{26}$ wetting of the bottom surface of the component starts upon contact with the liquid interface, and the liquid stops spreading when the meniscus reaches the edges of the component (Fig. 1, bottom row).

In the following subsections, we show how analytical descriptions of capillary forces can be derived from surface energy- and force-based models for the simple case where the liquid meniscus completely wets the receptor and the component. In Section 3.2 this assumption will be relaxed.

\subsection{Surface energy models}

The mechanics of capillary self-alignment can be understood as the consequence of the minimization of the system's excess free energy. Specifically, the surface energy of the liquid meniscus is minimized when its interfacial area is minimized. In a proper design of the system, this condition is satisfied when the edges of the component are aligned to those of the receptor. Assuming other energy components to be negligible (e.g., gravitational energy, for Bond number Bo $\ll 1$ ), the excess free energy is represented by the interfacial energy $E$ of the system

$$
E=\gamma A
$$

where $\gamma$ is the surface tension and $A$ is the total interfacial area of the liquid. Assuming to know the equilibrium shape of the liquid meniscus for each offset $\vec{x}_{0}=\left[\begin{array}{ll}x_{0} & y_{0} z_{0}\end{array}\right]$ of the component, the restoring force $\vec{F}=F\left(\vec{x}_{0}\right)$ can be calculated from the energy function $E=E\left(\vec{x}_{0}\right)$ by

$$
\vec{F}=-\vec{\nabla} E
$$

Considering the energies of all interfaces, eqn (1) is expressed as:

$$
E=\gamma A_{\mathrm{LG}}+\gamma_{\mathrm{LC}} A_{\mathrm{LC}}+\gamma_{\mathrm{LR}} A_{\mathrm{LR}}
$$

where $\gamma_{* *}$ and $A_{* *}$ represent respectively the interfacial energies and areas among liquid (L), gas (G), component (C) and receptor (R) (see Fig. 1b). In case of e.g. fluidic self-assembly, the gas phase can be replaced by the immiscible liquid hosting the process. ${ }^{27-29}$ Under the common and simplifying assumptions of perfect wetting (Section 2.2), which implies full and constant liquid coverage of the liquid-solid interfacial areas ( $A_{\mathrm{LC}}$ and $A_{\mathrm{LR}}$ ), and absence of surface tension gradients, eqn (2) reduces to:

$$
\vec{F}=-\vec{\nabla}\left(\gamma A_{\mathrm{LG}}\right)=-\gamma \vec{\nabla} A_{\mathrm{LG}}
$$

Eqn (4) can be used for estimating self-centering forces for simplified geometries of liquid menisci, such as given by circular $^{30,31}$ or rectangular $^{2,32,33}$ components on shape-matching receptors under purely lateral translation (Fig. 3). By approximating the shape of the corresponding menisci as a sheared cylinder and parallelepiped, respectively, the energy can be written as:

$$
\begin{aligned}
& E_{\text {circ }}=\gamma R h \int_{0}^{2 \pi} \sqrt{1+\cos ^{2} \theta \frac{x^{2}}{h^{2}}} \mathrm{~d} \theta \\
& E_{\text {rect }}=\gamma\left(2 c h+2 c \sqrt{h^{2}+x^{2}}\right)
\end{aligned}
$$

For small displacements $(x \ll h)$, the lateral force $F_{x}=-\mathrm{d} E / \mathrm{d} x$ can be approximated as:

$$
\begin{gathered}
\bar{F}_{\text {circ }} \approx-\pi R \gamma \frac{x}{h} \overline{1}_{x} \\
\bar{F}_{\text {rect }} \approx-2 c \gamma \frac{x}{h} \overline{1}_{x}
\end{gathered}
$$


(a)

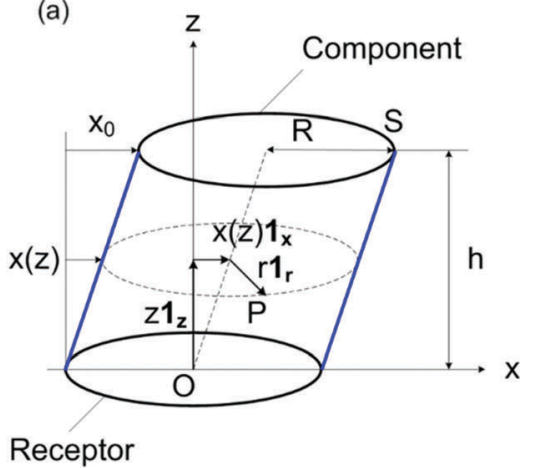

(b)

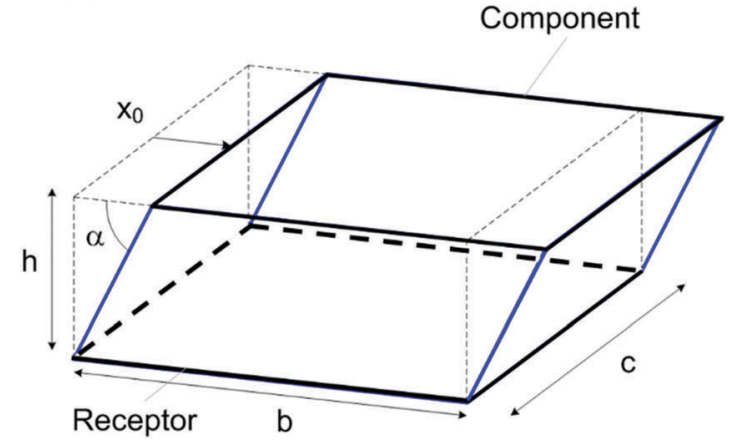

Fig. 3 Simplified meniscus geometries for the analytical derivation of the capillary force from the surface energy: case of a circular (a) and of a rectangular component (b) over shape-matching receptors.

that is, the lateral restoring force is linearly proportional to the displacement from equilibrium, as in an Hookean spring, and to (half of) the perimeter of the component/receptor. Following this approach, Berthier et al. generalized eqn (5)-(8) to the case of arbitrary polygonal components. ${ }^{34}$ Gao and Zhou proposed a unified semi-analytical model that describes small deformations of liquid menisci through plate series. ${ }^{35}$ It should be emphasized that these are only coarse approximations, since real menisci are not ideal cylinders nor parallelepipeds. More accurately, the energy $E$ has to be solved by finding the actual shape of the liquid meniscus under the given boundary conditions. In the most general cases, the solution can only be found computationally. This requires modelling the meniscus by digital tools, such as multiphysics software or Surface Evolver (ref. 2 and 8 provide extensive descriptions and examples). Numerical simulations additionally allow the characterization of the fluid geometries for all roto-translational degrees of freedom. ${ }^{4,32,36}$

\subsection{Capillary force models}

In the previous section, the capillary self-alignment process was analysed through energy minimization, and centering forces were derived through the energy gradient. The centering capillary forces can alternatively and equivalently be calculated directly from the composition of their two contributions: the tension force and the Laplace force. Indeed two distinct concepts merge under the denomination of capillary forces: the tensile, tension or surface tension force $F_{\mathrm{T}}$, and the pressure, Laplace or simply capillary force $F_{\mathrm{L}}$. The reader must bear in mind these two different physical effects, adding to one another to constitute what we will call the capillary force in the following.

The first contribution $F_{\mathrm{T}}$ is due to the tensile force exerted by the liquid meniscus on the solid and directed along the tangent to the liquid-gas interface. The surface tension force, always tensile, is quantified by the integral of the surface tension along the triple contact line (see Fig. 4 for symbols definition):

$$
\bar{F}_{\mathrm{T}}=\int \gamma \mathrm{d} l \overline{1}_{\mathrm{t}}
$$

According to recent studies by Marchand et al. ${ }^{37}$ this is to be considered an imprecise physical description of the capillary

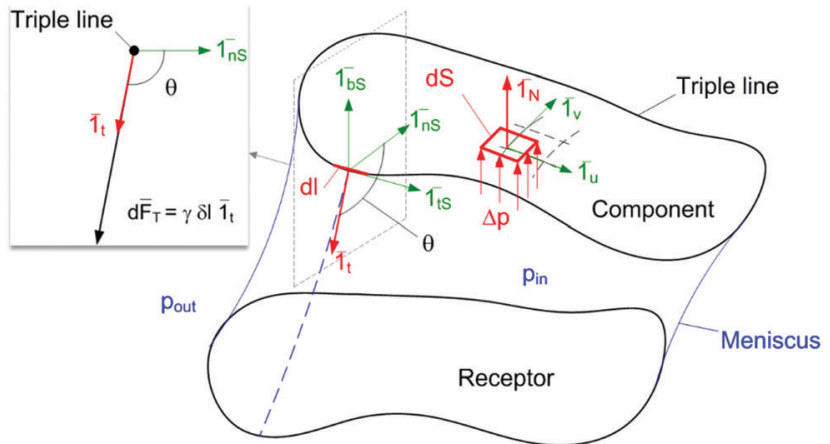

Fig. 4 Free body diagram of the component showing the tensile and pressure components of the capillary force. The tensile force is computed as the line integral of the force element $\mathrm{d} \bar{F}_{\mathrm{T}}=\gamma \mathrm{d} / \overline{1}_{\mathrm{t}}$ (see inset). The Laplace force is computed as the surface integral of the force element $\mathrm{d} \bar{F}_{\mathrm{L}}=$ $2 H \gamma d S \overline{1}_{N}$ where, according to the depicted sketch, $\Delta p=2 H \gamma$ is negative. Unit vectors are defined in the inset.

effect of a liquid on a compliant solid. We will however use the traditional description in the following, since both theories agree with one another for rigid solids.

The second effect is associated with the so-called Laplace pressure drop $\Delta p$, induced by the curvature of the meniscus. ${ }^{38}$ The Laplace pressure drop is given by the well-known YoungLaplace equation:

$$
p_{\text {in }}-p_{\text {out }}=\Delta p=2 H \gamma
$$

where $2 H$ is the total curvature, equal to the sum of the orthogonal curvatures $1 / \rho_{1}$ and $1 / \rho_{2}{ }^{2,22}$ This leads to a pressurerelated force $F_{\mathrm{L}}$ equal to:

$$
\bar{F}_{\mathrm{L}}=\iint \Delta p \mathrm{~d} S \overline{1}_{\mathrm{N}}
$$

These concepts are exemplified in the snapshot shown in Fig. 5. In this case the surface tension force is estimated to equal the perimeter of the square chip $(800 \mu \mathrm{m})$ times the surface tension of water $\left(72 \mathrm{mN} \mathrm{m}^{-1}\right.$ at room temperature) projected along the symmetry axis by an angle of $10^{\circ}$, i.e. $F_{\mathrm{T}}=59 \mu \mathrm{N}$ along the diagonal defined by $-\left(\overrightarrow{1}_{x}+\overrightarrow{1}_{y}\right) / \sqrt{2}$. Estimating the radii 

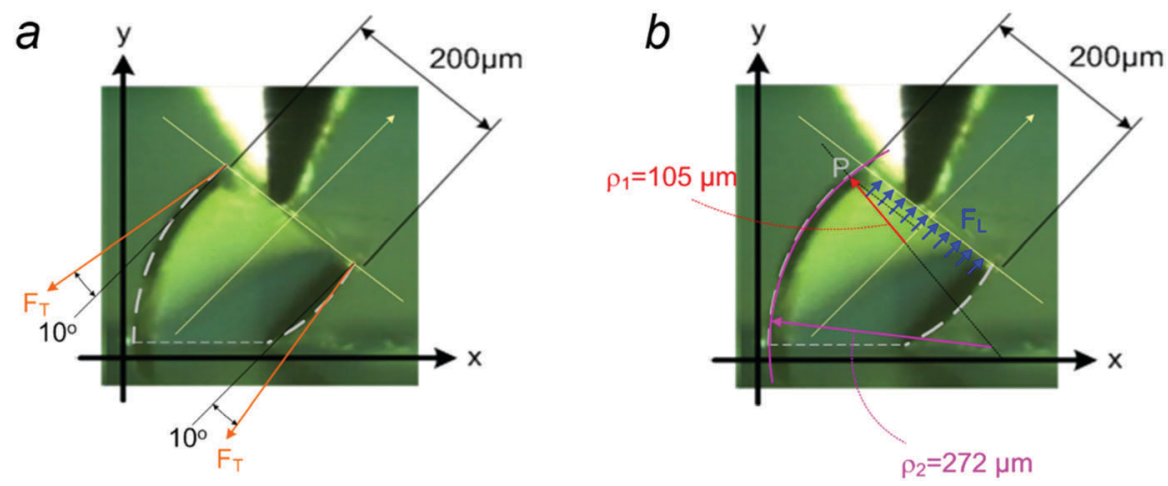

Fig. 5 Force analysis on the capillary self-alignment study described in ref. 39. (a) Estimation of surface tension force, (b) estimation of Laplace pressure effects (adapted from ref. 39, (c) 2012, American Institute of Physics).

of curvature to be $\rho_{1}=105 \mu \mathrm{m}$ and $\rho_{2}=272 \mu \mathrm{m}$ leads to a curvature $2 H=13200 \mathrm{~m}^{-1}$, and a Laplace pressure $\Delta p \cong 950 \mathrm{~Pa}$. This provides a Laplace force $F_{\mathrm{L}}=38 \mu \mathrm{N}$ along the diagonal $+\left(\overrightarrow{1}_{x}+\overrightarrow{1}_{y}\right) / \sqrt{2}$. The resulting capillary force is $F_{\mathrm{T}}+F_{\mathrm{L}}=21 \mu \mathrm{N}$ along the diagonal $-\left(\overrightarrow{1}_{x}+\overrightarrow{1}_{y}\right) / \sqrt{2}$.

\subsection{Model equivalence}

The modelling methods presented in Section 2.3 (energetic) and 2.4 (sum of Laplace and tension force components) are demonstrably equivalent. As a quick illustration, let us consider the configuration of Fig. 3b. The Laplace pressure acts perpendicular to the bottom surface of the component and does not contribute to the lateral restoring force along $x$. The tension force only contributes to the total capillary force along $x$, and can be expressed as:

$$
\bar{F}_{\text {rect }} \approx 2 c \gamma \cos \alpha \overline{1}_{x}
$$

where $\cos \alpha=x / \sqrt{x^{2}+h^{2}} \approx x / h$ for $x \ll h$, leading to $\bar{F}_{\text {rect }} \approx-2 c \gamma x / h \overline{1}_{x}$ as in eqn (8).

A more detailed analysis of the equivalence of energy- and force-based models is reported in ref. 40 .

\section{Liquid confinement}

Liquid confinement amounts to imposing a barrier to the spreading of a liquid on a substrate. To be localized on the target substrate, a liquid droplet must be confined along its entire perimeter by the edges of the receptor. Without confinement of the liquid meniscus, the final position of the component is underspecified and not predictable. Only in presence of liquid confinement can the shape of the receptor conveniently match the profile of the component.

\subsection{Receptor types and fabrication}

Liquid confinement on a solid substrate can be enforced in a surface-chemical way, in a geometrical way, or in a combination thereof. $^{26,41}$ In all cases, the mechanism underlying liquid confinement is the pinning of the contact line and the associated extension of the contact angle hysteresis along the pinned perimeter of the droplet (Section 2.2).

The surface-chemical method (Fig. 6a) exploits the wetting contrast between the interior surface of a receptor (i.e., the receptor proper) and the exterior surface, which can be intended e.g. to space arrays of receptors apart according to a defined pitch. ${ }^{42}$ The receptor surface needs to be significantly more wettable by the liquid than the spacer surface.
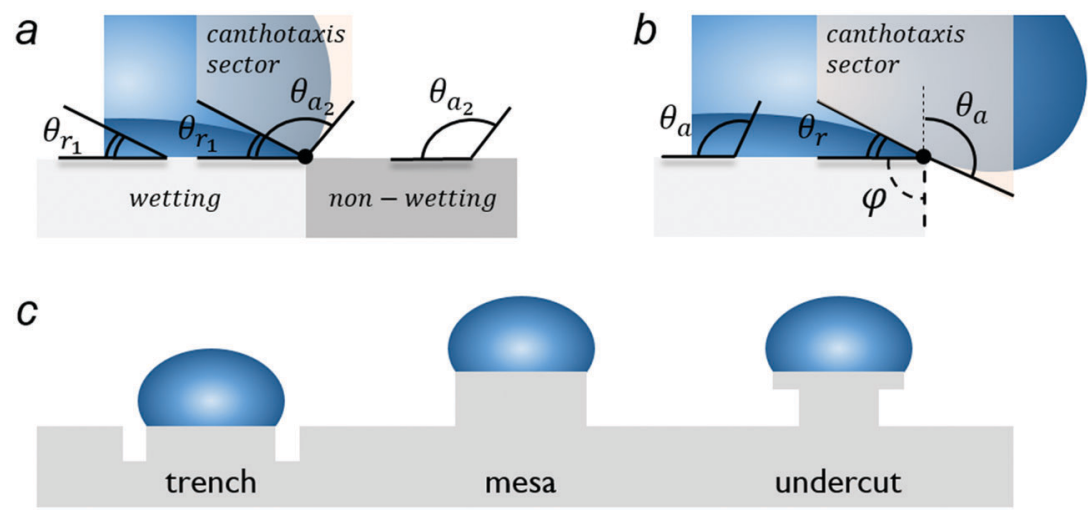

Fig. 6 Liquid confinement within receptors. (a) Surface-chemical confinement, exploiting the wetting contrast between adjacent surfaces. (b) Topographical confinement, using the geometry of a solid edge of aperture $\varphi$. (c) Implementations of topographical liquid confinement. 

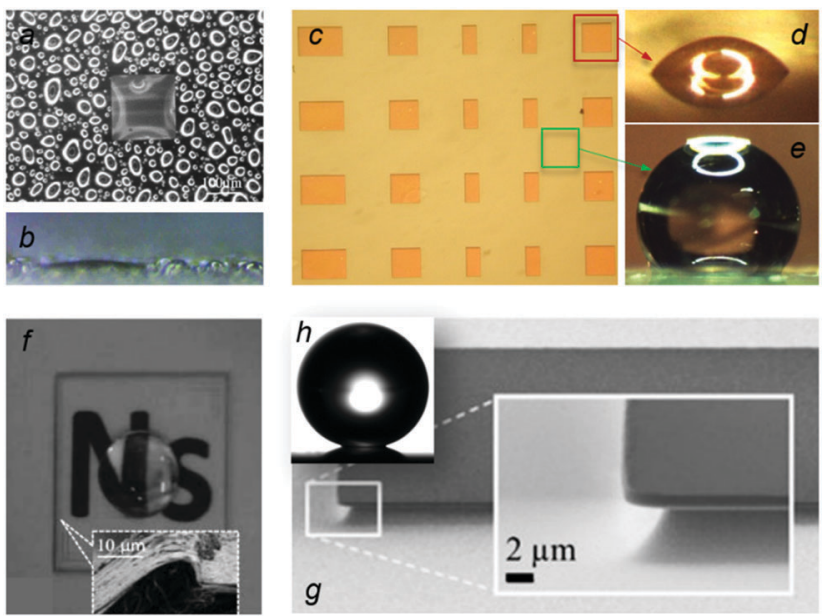

Fig. 7 Microfabricated receptors for surface chemical (a-e) and topographical $(f-h)$ liquid confinement. (a) Top view and (b) side view of a hydrophilic silicon dioxide receptor surrounded by hydrophobic fluorocarbon-treated spacer. Water droplets wet only the hydrophilic receptor (adapted from ref. 54, (C) 2011, Institute of Physics). (c) Oleophilic Au-coated receptors surrounded by oleophobic porous spacer functionalized with a fluorinated self-assembled monolayer (adapted from ref. 45, (C) 2011, American Institute of Physics). (d) Side view of a droplet of oillike adhesive on $\mathrm{Au}$, and (e) droplet of the same adhesive on the background (adapted from ref. 45, (C) 2011, American Institute of Physics). (f) Topographical confinement of a water droplet within a laser-scribed receptor on plastic substrate (adapted from ref. 55, courtesy of G. Arutinov). (g) SEM micrograph of a receptor topographically defined by undercut edges (reprinted with permission from ref. 56, (C) 2013, IEEE). (h) An oillike adhesive droplet confined on a receptor defined by an undercut edge, showing $\sim 180^{\circ}$ apparent contact angle (adapted with permission from ref. 49, (c) 2013, Wiley).

The difference between the liquid contact angles on the adjacent surfaces provides a rough quantification of the wetting contrast. According to this metric, a difference larger than $60^{\circ}$ is sufficient for robust chemical confinement. ${ }^{42-44}$ Once on the edge of the receptor, the contact line remains pinned for all values of the edge angle comprised within the canthotaxis sector, delimited respectively by the receding contact angle on the wetting side and by the advancing contact angle on the non-wetting side. ${ }^{8}$ Hydrophilic (Fig. $7 \mathrm{a}$ and b) or oleophilic receptors (Fig. 7c-e) are specifically implemented to be highly wettable by water or hydrocarbons, respectively. Chemical confinement is effective also for liquids of low surface tension in air. ${ }^{45}$

The geometrical or topographical method makes use of sharp solid edges of the substrate to define the shape of the receptor $^{46}$ (Fig. 6b). In this case, the edge angle of the liquid needs to increase up to the advancing contact angle value with respect to the plane tangent to the outer side of the receptor before the contact line can further advance, causing liquid overflow out of the site. Such extension of the contact angle hysteresis is captured by the so-called Gibbs' criterion, which for a chemically-homogeneous solid surface defines the canthotaxis sector as $\left[\theta_{\mathrm{r}}, \theta_{\mathrm{a}}+\pi-\varphi\right]$, where $\varphi$ is angle spanned by the solid edge. ${ }^{47}$ The most common geometries of topographical receptors include trench, ${ }^{48}$ mesa and undercut types (Fig. 6c and 7f, g). Notably, by using receptors with solid undercuts, it is possible to confine low surface tension liquids in air, and obtain apparent contact angles in excess of $180^{\circ}$ (Fig. 7h). ${ }^{49}$ Sparse arrays of micropillars with a doubly-reentrant nanomachined tip geometry were recently demonstrated to turn every solid surface, including highly wetting ones, superrepellent to all liquids. ${ }^{50}$

An issue for mesa-type receptors of micrometric size is the required step height difference, which should be of the order of $1 \mu \mathrm{m} .{ }^{51}$ Receptors exploiting a hydrophilic/hydrophobic wetting contrast are relatively easy to fabricate using photolithography. Conversely, the more reliable hydrophilic/superhydrophobic contrast is challenging to enforce due to the surface nanoroughness required in the spacer. Recently, electron beam was employed to achieve patterned wetting and create $\mu \mathrm{m}$-sized superhydrophilic patterns on superhydrophobic substrates. ${ }^{52}$ Hydrophobic sidewalls surrounding hydrophilic mesa-type receptors were also shown to improve liquid confinement and benefit the capillary self-alignment performance. ${ }^{26,41}$ Similar observations were shown to apply to the surface conditioning of components. ${ }^{53}$

\subsection{Partial wettability}

In presence of perfect wetting $(\theta \rightarrow 0$, Section 2.2$)$, the contact lines of the liquid meniscus are pinned along the edges of both receptors and components. The perfect wetting condition represents a useful model simplification and a good approximation for common applications, such as e.g. solder bumps over oxide-free metal pads or water on highly hydrophilic receptors (see previous section). However, perfect wetting does not capture the most general scenario. ${ }^{57}$

Upon dispensing, a large advancing contact angle may prevent the liquid to wet the complete receptor. Capillary selfalignment was nonetheless demonstrated also on partially wetting or hydrophobic receptors, such as e.g. with advancing and receding water contact angles of $118^{\circ}$ and $69^{\circ}$, respectively. ${ }^{39}$ Wetting of the receptors was in this case forced by using excessive amounts of liquid or by applying pressure on the component. Capillary self-alignment across relatively large component offsets is another significant instance where partial wetting affects the performance of the process in terms of accuracy and yield. ${ }^{48}$ Partial wetting makes the liquid/solid interfacial areas time-variant during transient component motion ${ }^{26,57}$ and, as expected from eqn (3), this affects lateral restoring forces. ${ }^{58} \mathrm{~A}$ recent piecewise model of lateral restoring capillary forces can justify the experimental observations by accounting for partial liquid wetting. ${ }^{33}$ According to what was mentioned in Section 2.2, in this case the boundary conditions must be adapted to the offset of the component to reproduce the local meniscus geometry in proximity of the contact lines. In the model, progressively larger lateral deformations of the liquid meniscus, corresponding to progressively larger component offsets, are accommodated by switching the boundary conditions from pinned contact line to prescribed receding contact angles. ${ }^{33}$ 


\section{Influence of shape and size}

Capillary self-alignment typically requires the shape of the component to be commensurate with that of the receptor. The general design guideline is that, for a given initial component offset, the liquid meniscus constrained by receptor and component (or a part of it) possesses sufficiently higher potential energy compared to its equilibrium shape so that its relaxation can sustain the self-aligning motion, as discussed in Section 2. Most reported instances of the process target perfect final alignment between the components and the receptor. Accordingly, components and receptors have shapes and sizes matching up to the degree allowed by fabrication process, ${ }^{29,48,54,59-61}$ which is often in the order of fractions of $\mu \mathrm{m}$. In some applications, the receptor is fractioned into several separated parts, due to the requirements of electrical functionality, structure of the device, or to remove excessive amount of liquid after alignment. ${ }^{62,63}$ The working principle for such single-component to multi-receptor embodiment is analogous to the standard single-component to single-receptor self-alignment configuration, except for the mechanism of meniscus formation, which may slightly differ due to the segmented shape of the receptor. ${ }^{62}$

\subsection{Capillary length and liquid volume}

The volume of the liquid meniscus is an important parameter for achieving high yields from the self-alignment process. To discuss the appropriate liquid volume for self-alignment, the concept of capillary length $L_{\mathrm{C}}=\sqrt{\gamma / \rho g}$ is useful, where $\gamma$ is the surface tension of the droplet, $\rho$ is the density of the liquid and $g$ is the gravitational constant. For a water droplet in air at room temperature, the capillary length is about $2.7 \mathrm{~mm}$. Capillary length can be used to quickly check the dominating force in self-alignment regarding the different size of patterns. For example, when the components and receptors are smaller than the capillary length, and so is the gap, it is safe to say that the surface tension will dominate the self-alignment process. Similarly, by replacing the gravitational constant $g$ by an acceleration, an equivalent cut-off length can be defined, which is relevant for high-acceleration pick-and-place machines. ${ }^{6}$

However, $L_{\mathrm{C}}$ alone cannot be used to determine whether selfalignment will succeed or not. Other important parameters are volume and corresponding thickness of the meniscus between component and receptor, surface wetting, the weight of the component and the possibility of friction between solid surfaces. Self-alignment of rather thin, $20 \mathrm{~mm}$-sized components (i.e. an order of magnitude larger than $L_{\mathrm{C}}$ of water) was reported with good success rates..$^{20,48,64,65}$ Even below the capillary length and in absence of liquid overflow, an excessive liquid volume can cause a tilted and misaligned component pose, corresponding to a locally stable meniscus geometry. ${ }^{66,67}$ Conversely, at the small droplet limit there is not enough liquid to wet both bounding surfaces, the component can touch the substrate and dry friction can prevent alignment completion.

For maximizing yield of self-alignment, there may be an optimum liquid volume value between these extremes. To the knowledge of the authors, only a few design rules for selecting the droplet volume have been provided. ${ }^{67}$ For the specific case of $300 \mu \mathrm{m} \times 300 \mu \mathrm{m}$ cuboidal components assembled over square shaped receptors, the optimal droplet volume maximizing yield was experimentally found between 1.8 and $2.7 \mathrm{~nL},{ }^{60}$ whereas a gap of $125 \mu \mathrm{m}$ was suggested to optimize the performance in the alignment of millimetre-sized foil dies. ${ }^{64}$ The addition of wetting bands around the edges or corners of the receptors was suggested to help avoid tilting of the components. ${ }^{66}$

\subsection{Shape matching}

Self-alignment does not require the shape of the components and of the receptors to strictly match each other. In early studies, ${ }^{28}$ the receptors were designed with shapes supposed to allow robust correction of initial angular offsets, such as halfdiscs and commas. The patterns could not exclude unintended component placements corresponding to local energy minima. Analytical studies on the design of component and receptor shapes were pursued by Böhringer $e t a l{ }^{68,69}$ They developed a simple geometrical model to predict the energy landscape for the self-alignment of components and receptors based on the convolution of their shapes. The model is valid for arbitrary geometries and for large biases compared to the gaps. The model recovers the intuition according to which the energy of the system is smallest for the largest overlap between component and receptor, and is helpful to predict and avoid configurations associated with local energy minima. ${ }^{69}$

For the widely used cuboid-shaped components and square or rectangular receptors, capillary self-alignment faces several application scenarios depending on the feeding methods (Section 6.1). Particularly, if the shapes and sizes of the component and the receptor are significantly mismatched, besides local energy minima there can exist multiple relative positions which cannot be distinguished by their energy. In presence of such degeneracy, the final position is contingent to the details of feeding and can be hardly predicted with precision.

Fig. 8 shows general scenarios wherein the size of the components and of the receptors are significantly different and their final relative position is underconstrained. Cases $\mathrm{A}_{1}-\mathrm{B}_{3}$ represent a component placed onto a larger receptor. When released partially outside the receptor, the component can still marginally align to the receptor, provided that the motion is overdamped. This can lead to partial self-alignment $\left(A_{1}\right)$, corner self-alignment $\left(A_{2}\right)$ or edge self-alignment $\left(A_{3}\right) .^{70,71}$ The component may conversely overshoot past the receptor edge and pull the contact line with it, away from the edge, when small liquid viscosity and/or large liquid volumes induce underdamped motion. Insufficiently large offsets may also not be conducive to the alignment conditions $A_{1}-A_{3}$. In these cases, the component may end up in either of the $B_{1}-B_{3}$ configurations $\left(B_{2}\right.$ being equivalent to $A_{3}$ ). When the weight of the component is insignificant compared to surface tension of the meniscus, the relative sizes of components and receptors can also be inverted, so that for instance the configurations $\mathrm{C}_{1}-\mathrm{C}_{3}$ can be mapped onto $A_{1}-A_{3}$. It is interesting to note that in all cases, except for $B_{3}$, the in-plane orientation of the component will be corrected during the self-alignment. 



Fig. 8 Scenarios for capillary self-alignment where the sizes of component and receptor are not commensurate. $\left(A_{0}\right)$ Default: component and receptor with matching shapes; $\left(A_{1}\right)$ matching of a single lateral size, leading to partial self-alignment; $\left(A_{2}\right)$ component matching the shape of a corner of the receptor, leading to corner self-alignment; $\left(A_{3}\right)$ component matching the shape of an edge of the receptor, leading to edge self-alignment. ( $\left.B_{1}-B_{3}\right)$ Components released from undesired offsets, leading to poor self-alignment or even no alignment. $\left(C_{1}-C_{3}\right)$ Reciprocal cases to $A_{1}-A_{3}$, leading to similar results only in absence of component tilting and of friction between dry surfaces.

Capillary self-alignment can also be used to align components with more sophisticated geometries than the primary shapes previously analysed. ${ }^{72}$ Millimeter-sized MEMS force sensors were picked up and precisely aligned to a pre-patterned printed circuit board (PCB). ${ }^{73}$ The capillary force of molten solder bumps between matching pads on both board and sensors was exploited, and three devices were assembled simultaneously. A capillary approach was also applied to the fluidic assembly of a GaAs-based microcantilever spin injector, ${ }^{70}$ microcantilever for atomic force microscopy, ${ }^{71}$ and of millimeter-sized components with inner cavities. ${ }^{63}$ In this case the hydrophilic receptors, coated with a thin layer of water-diluted hydrofluoric acid, matched only the perimeter of the components. The receptors contained additional microchannels for liquid evacuation, so that an interior area free of liquid could safely be maintained to host additional structures. This solution is suitable for sealed packaging of e.g. sensors and MEMS devices.

When the size of the component and the receptor are commensurate, the self-alignment can allow accurate positioning despite mismatch in shape or edge profile (Fig. 9). Defects inside the receptor or along the edge of the receptor bear relatively little influence to the alignment. ${ }^{74}$ Even when the edge of the receptor is poorly defined, due to e.g. low quality manufacturing, the self-alignment will still lead to rather good accuracy. For example, for receptors of size $200 \mu \mathrm{m} \times 200 \mu \mathrm{m}$ with edge jaggedness of $\pm 4 \mu \mathrm{m}$, the lateral and orientational self-alignment accuracy of smooth matching components was $\pm 1 \mu \mathrm{m}$ and $\pm 0.6^{\circ}$ respectively, which is significantly better than the definition of the pattern. The reason for this is that the energy minimization of the self-alignment process arises from a weighted average

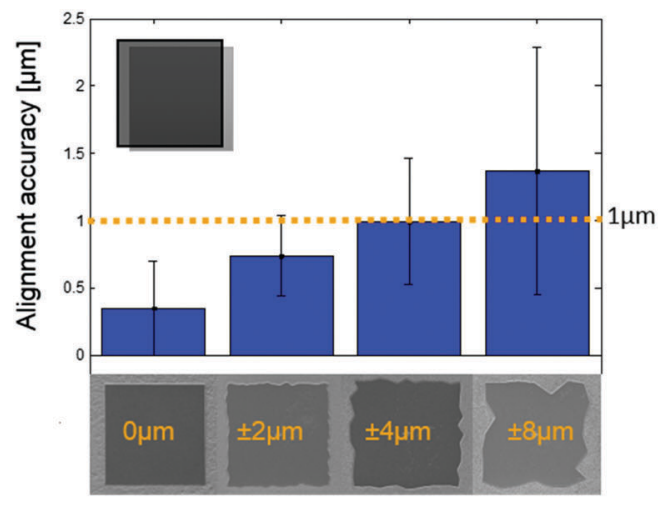

Edge jaggedness

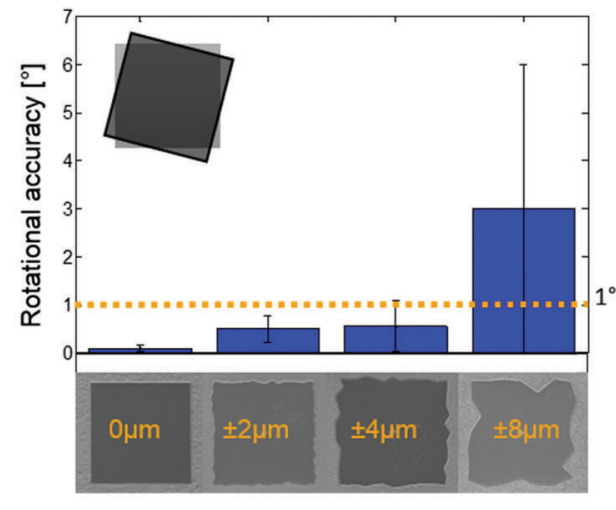

Edge jaggedness

Fig. 9 The alignment accuracy of $200 \mu \mathrm{m} \times 200 \mu \mathrm{m} \times 50 \mu \mathrm{m}$ components on $200 \mu \mathrm{m} \times 200 \mu \mathrm{m}$ receptor with different edge profiles: linear, jaggedness of $\pm 2 \mu \mathrm{m}, \pm 4 \mu \mathrm{m}$ and $\pm 8 \mu \mathrm{m}$ (adapted from ref. 74, (C) 2014, IEEE). 
where the influences of opposite edge distortions may cancel each other out, leading to a modest magnitude of the total error.

\subsection{Size of components}

Capillary self-alignment can be applied to a large range of component sizes, from tens of $\mu \mathrm{m}$ to tens of $\mathrm{mm}$, and to components with length to thickness ratio up to 100 .

4.3.1 Large components. Capillary self-alignment works best when surface tension dominates. When the characteristic size of the component is larger than a few $\mathrm{mm}$, gravitational and inertial effects may become comparable (Section 4.1). Nevertheless, self-alignment of relatively large dies has been successfully demonstrated. In this case, the thickness of the dies is usually below $1 \mathrm{~mm}$, and the height of the liquid meniscus measures few hundreds $\mu \mathrm{m}$, such that capillary forces still prevail.

Assembly of arrays of $20 \mathrm{~mm}$ dies on carrier substrates through a combination of parallel vacuum gripping and capillary self-alignment was reported. ${ }^{65}$ The capillary self-alignment of 10 to $20 \mathrm{~mm}$-sized plastic foil dies fed by pick-and-place onto silicon-based $^{64}$ and laser-scribed plastic substrates ${ }^{48}$ was also demonstrated, the latter being also suitable for autonomous deployment onto moving webs. ${ }^{75}$ Centimeter-sized components floating at the air/water interface were assembled onto matching hydrophilic receptors of hydrophobic substrates. ${ }^{76}$ The substrate was pulled at an angle through the fluid interface, and unique pre-orientation of the components could also be enforced by using a magnetic template. ${ }^{77}$

Capillary self-alignment is also simplifying 3D microelectronic integration. For instance, several layers of $5 \mathrm{~mm} \times$ $5 \mathrm{~mm} \times 50 \mu \mathrm{m}$ chips with $5 \mu \mathrm{m}$-diameter through-silicon VIAs (TSVs) were self-aligned and stacked onto BEOL-processed silicon substrates. ${ }^{19}$

4.3.2 Ultra-small components. $20-60 \mu \mathrm{m}$-sized semiconductor components were assembled on a vertically pulled substrate at a silicon oil/water/substrate triple interface. ${ }^{59,78}$ The surface chemical treatment of the components and the chosen immiscible fluid couple made the trapping of the chiplets at the interface energetically favourable. The authors reported the largest self-assembly throughput to date with 62500 chips assembled in $45 \mathrm{~s}$, equivalent to $1388 \mathrm{~s}^{-1}$. The process requires significant preparation steps for each assembly run, and repeated crossing of the substrate surface by the liquid interface carrying the chiplets is needed to achieve the claimed high surface coverage of the receptors. The reported accuracy and assembly yield were $0.9 \mu \mathrm{m}$ and $98 \%$, respectively.

4.3.3 Ultra-thin components. Capillary self-alignment can be particularly suitable to handle very thin and fragile components, since gravity is small for the otherwise significant size of the chips. 12 ultra-thin $10 \mu \mathrm{m}$ chips were successfully stacked and precisely aligned on top of each other using robotic pick-and-place and capillary self-alignment. ${ }^{79}$ Capillary forces were also combined with magnetic alignment, mediated by magnetically-susceptible metal interconnects, for the templated integration of $20 \mu \mathrm{m}$-thick components onto polymer foils. ${ }^{80}$

\section{Dynamics}

\subsection{Inertial and viscous effects}

A no-flow situation was implicitly considered in the previous sections, with the liquid meniscus at mechanical equilibrium. To describe the physics of the process in presence of liquid flow in the meniscus, the associated Navier-Stokes equations must be solved. The viscous effects induced by the flow can then be compared to the capillary effects through the capillary number $\mathrm{Ca}=\frac{\mu \nu}{\gamma}$, while the relative magnitude of inertial over viscous effects is considered in the Reynolds number $\operatorname{Re}=\frac{\rho v L}{\mu}$, with $L$ the characteristic dimension of the system (typically, the component sidelength). The comparison of capillary, viscous and inertial effects was studied by Valsamis et al. in the case of axial motion of liquid bridges, ${ }^{81}$ illustrating the existence of three distinct regimes: a capillary regime for small $\mathrm{Ca}$ and small $\mathrm{Re}$, a viscous regime for large $\mathrm{Ca}$ and small $\mathrm{Re}$, and an inertial regime otherwise. It remains unknown whether this description is valid also for lateral motion.

To go beyond pure scaling laws, it is necessary to consider the set of Newton and Navier-Stokes equations describing the alignment process. For the simplified cylinder and parallelepiped geometries of Fig. 3, where only the lateral displacement of the component during the self-alignment motion is considered, it is possible to develop a simplified analytical approximation of the viscous force ${ }^{82}$ by assuming Couette flow between the component and the receptor. Assuming a linear velocity profile, the viscous shear stress on the component can be written as $\tau=\mu \frac{\partial v_{x}}{\partial y} \approx \mu \frac{\dot{x}}{h} \approx \mu \frac{1}{h} \frac{\mathrm{d} x}{\mathrm{~d} t}$, and the viscous force is thus equal to:

$$
F_{\text {viscous }}=-\frac{\mu A_{\mathrm{LC}}}{h} \frac{\mathrm{d} x}{\mathrm{~d} t}
$$

where $\mu$ is the dynamic viscosity, $A_{\mathrm{LC}}=w l$ and $w, l, x$ and $h$ are respectively the component width, length, offset and the gap. For the parallelepiped case of Fig. 3b, this can be combined with Newton's law and the linear approximation of the surfacetension force from eqn (8) to describe a damped second-order dynamics of the capillary self-alignment process:

$$
m \frac{\mathrm{d}^{2} x}{\mathrm{~d} t^{2}}+\frac{\mu w l}{h} \frac{\mathrm{d} x}{\mathrm{~d} t}+\frac{2 \gamma w x}{h}=0
$$

From this model, one can estimate the natural frequency $\omega$ and the damping coefficient $\xi$ of the dynamical system:

$$
\begin{gathered}
\omega=\sqrt{\frac{2 \gamma w}{h m}} \\
\xi=\frac{\mu l w}{\sqrt{8 \gamma m w h}}
\end{gathered}
$$

These results can thus be interpreted as usual for a secondorder system dynamics.

The linear velocity assumption used above only holds when the flow velocity has the time to develop a linear profile, i.e. after a timescale of about $h^{2} / \nu, \nu$ being the kinematic viscosity. 


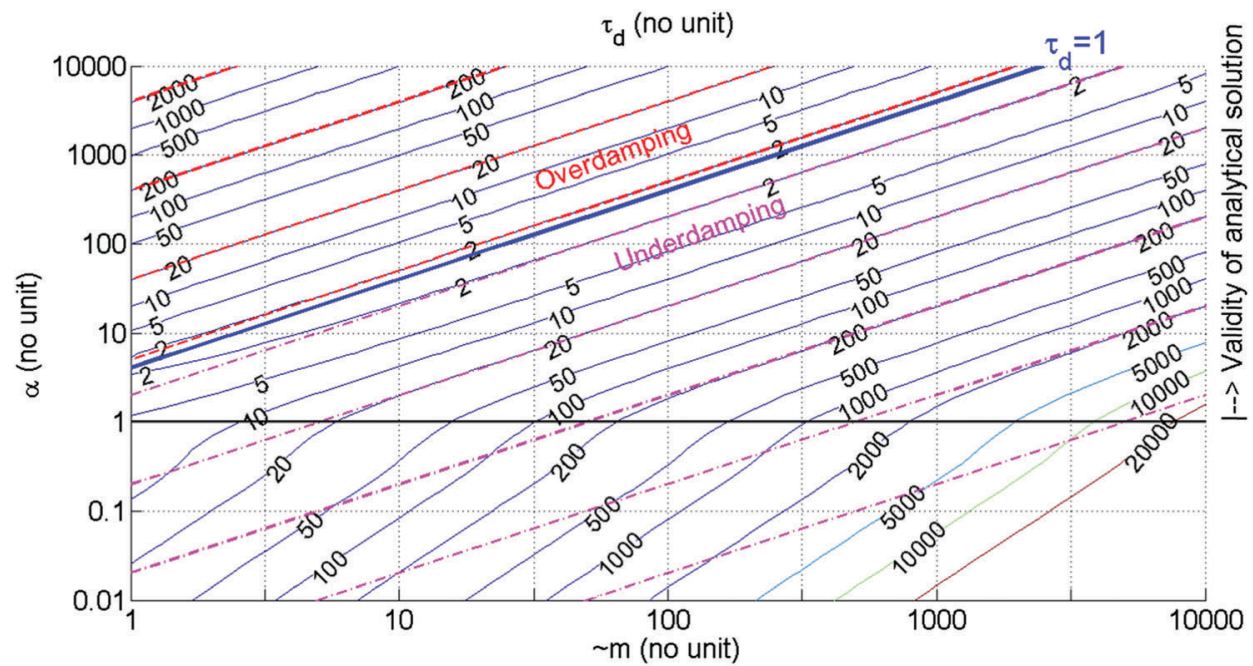

Fig. 10 Non-dimensional characteristic time $\tau_{\mathrm{d}}$ as a function of non-dimensional viscosity $\alpha$ and mass $\tilde{m}$. The stripped lines indicate the analytical solutions to eqn (12) of the linear second-order system, valid for $\alpha>1$. For smaller $\alpha=\frac{4 \nu \tau}{h^{2}}$, the solid lines are obtained from numerical simulations $s^{30}$ and deviate from the analytical expression [reproduced from ref. 2, (C) Springer, 2013].

If the restoring capillary force triggers oscillations with a shorter period, which can be the case for large gaps or low viscosities, the coupled equations must be solved simultaneously. ${ }^{30,83}$ The results are represented in Fig. 10, where $\tau=\sqrt{m / k}$ is a characteristic time of the oscillations of the component mass $m$ subjected to a capillary stiffness $k$ (e.g. for rectangular components, $\left.k=\frac{2 c \gamma}{h}\right), \alpha=\frac{4 \nu \tau}{h^{2}}=\frac{4}{\mathrm{Re}}$ is a non-dimensional viscosity (corresponding to 4 times the ratio of the characteristic time $\tau$ and the timescale $\left.h^{2} / \nu\right), \tilde{m}=\frac{2 m}{\rho w l h}$ is a non-dimensional mass (component mass scaled by the liquid mass). The map then gives the non-dimensional characteristic time $\tau_{\mathrm{d}}=\frac{\tau_{\mathrm{c}}}{\tau}$ associated to the second-order system defined above, where the characteristic time $\tau_{\mathrm{c}}=\frac{2 m h}{\mu w l}$ when eqn (13) holds. Table 1 lists pertinent values of $\mathrm{Ca}$ and Re extracted from recent literature.

The in-plane modes of oscillation of a millimeter-sized selfaligning component were recently tracked simultaneously and with high spatial and temporal resolution by means of a highspeed camera (Fig. 1, bottom row). ${ }^{20}$ The study evidenced the possibility of both uncoupled and coupled motion along the degrees of freedom of the fluid joint depending on the initial conditions of component release. As noted above, increased viscosity and lower surface tension slow down the capillary

Table 1 Capillary and Reynolds numbers from selected recent works

\begin{tabular}{llll}
\hline Reference & Liquid & Ca & Re \\
\hline Lu and Bailey $(2005)^{83}$ & Solder paste & $4.62 \times 10^{-5}$ & 0.933 \\
Lambert et al. $(2010)^{30}$ & Glue Dymax & 0.012 & 0.055 \\
Lambert et al. $(2010)^{30}$ & Silicone oil & 0.158 & 0.010 \\
Lambert et al. $(2010)^{30}$ & Silicone oil & 75404 & 0.002 \\
Arutinov et al. $(2014)^{20}$ & Water & $1.39 \times 10^{-6}$ & 0.013 \\
Dubey et al. $(2016)^{84}$ & Water & $1.22 \times 10^{-4}$ & 0.674
\end{tabular}

self-alignment process, making it easier to visualize using ordinary frame rate cameras.

The full dynamics of chip alignment is based on the coupling of the 6 degrees-of-freedom of the component ( 3 translations and 3 rotations) with the flow dynamics of the meniscus. This amounts to coupling Newton's law for the component with Navier-Stokes equations for the fluid. Analytical estimates for the axial motion ${ }^{81,82}$ and, under strong assumptions, for the lateral motion ${ }^{82}$ were reported. This is however not representative for the transient period typical of the oscillatory motion of a self-aligning component, as observed by Arutinov et $a .^{20}{ }^{20}$ Other solutions were proposed by Lu and Bailey ${ }^{83}$ and Lambert et al. ${ }^{30}$ relying on the assumption of one-dimensional liquid flow, i.e., of liquid flowing only parallel to the fluid film $\left(v_{\|} \neq 0, v_{\perp}=0\right)$. None of these approaches however manages to describe very accurately the dynamics of capillary selfalignment of the component recorded experimentally. ${ }^{20}$

\subsection{Viscosity and surface tension of common liquids}

The previous analyses show that several physical and chemical parameters of the liquid menisci influence the self-alignment process: surface tension (typically ranging from some tens of $\mathrm{mN} \mathrm{m}^{-1}$ for water-based solutions, adhesives and oils up to several hundreds of $\mathrm{mN} \mathrm{m}^{-1}$ for molten metallic alloys), dynamic viscosity (ranging over several orders of magnitude) and density. For the most commonly used liquids we specifically report in Fig. 11 the values of surface tension and viscosity, which are explicitly involved in the analytical models (Section 2.3). It is apparent that the liquids can be grouped into two main clusters at opposite ends of the ranges, and that water sits roughly in between. It is also apparent that the viscosity varies up to 4 orders of magnitude between liquids, while the surface tension varies only over 1 order of magnitude. As seen in the previous section, the capillary number and the characteristic time constant are set by the ratio of viscosity and surface 


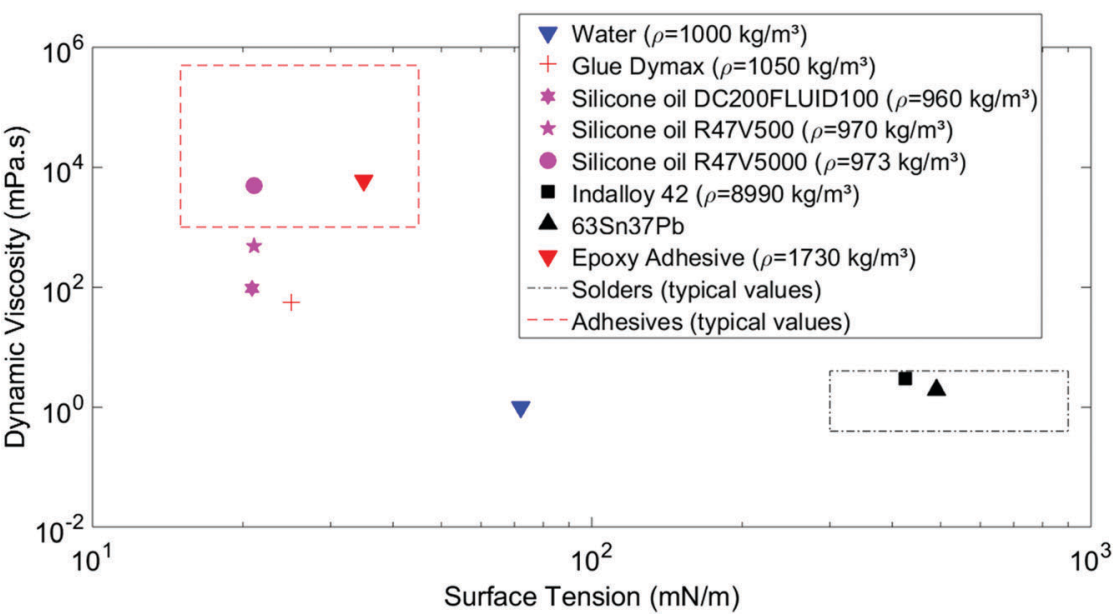

Fig. 11 Dynamic viscosity versus surface tension for most used liquids in capillary self-alignment. Typical orders of magnitude for adhesives and solders (rectangular boxes) are taken from ref. 85. Values for Dymax glue, silicone oil and molten alloy Indalloy can be found in ref. 30, and for epoxy adhesive in ref. 86. Liquid density values (needed to compute e.g. the Reynolds number, see Table 1) are indicated in the legend.

tension. Hence, since there is greater freedom in choosing liquid viscosity, to tune the self-alignment time it is usually preferred to change the viscosity of the liquid, all other parameters being equal.

\subsection{Experimental self-alignment dynamics}

As earlier mentioned, most of the works in capillary selfalignment have considered perfect wetting of liquids on both receptors and components (e.g., oxide-free molten solders on metal pads, water on oxidized surfaces), and excluded contact line unpinning and partial wetting during the process. In presence of contact line pinning, contact angles affect the selfalignment process only for what concerns liquid confinement, since vanishing contact angles and small wetting contrast tend to make the confinement unreliable and not robust ${ }^{48}$ (as discussed in Section 3.2). Conversely, upon delivery of the component on top of the confined droplet, the contact lines are generally not pinned, and the meniscus transiently spreads to reach the edges of the component (Fig. 1, bottom row). The dynamics of selfalignment is then significantly affected by liquid spreading, and thus by the contact angles. ${ }^{58}$ Higher wettability (i.e., lower contact angles) was shown to induce faster aligning motion of the components, ${ }^{20,58}$ consistently with the predictions of energy-based models. ${ }^{33}$ However, self-alignment can still be achieved through the relatively slow spreading of a liquid medium of high viscosity and low surface tension, such as resin materials ${ }^{86,87}$ or adhesives. ${ }^{29,45}$ Fig. 12 illustrates the capillary self-alignment between two shape-matching and vertically stacked $300 \mu \mathrm{m} \times 300 \mu \mathrm{m}$ SU-8 chips bridged by an adhesive of high viscosity curable by ultra-violet (UV) radiation. The process reached successful completion about 4 minutes after release of the top chip.

\section{Process integration}

The self-alignment process includes several essential process steps: component feeding, liquid deposition, self-alignment and post-processing. The order of component feeding and liquid deposition is determined by the design of the whole process.

\subsection{Liquids}

6.1.1 Liquid deposition and choice. To achieve surface tension-driven self-alignment, an appropriate volume of liquid must be introduced at proper time during the process. Liquids are conveniently introduced onto the receptors (see Section 3) and, especially in presence of very small volumes of volatile liquids, the component should be introduced shortly after.
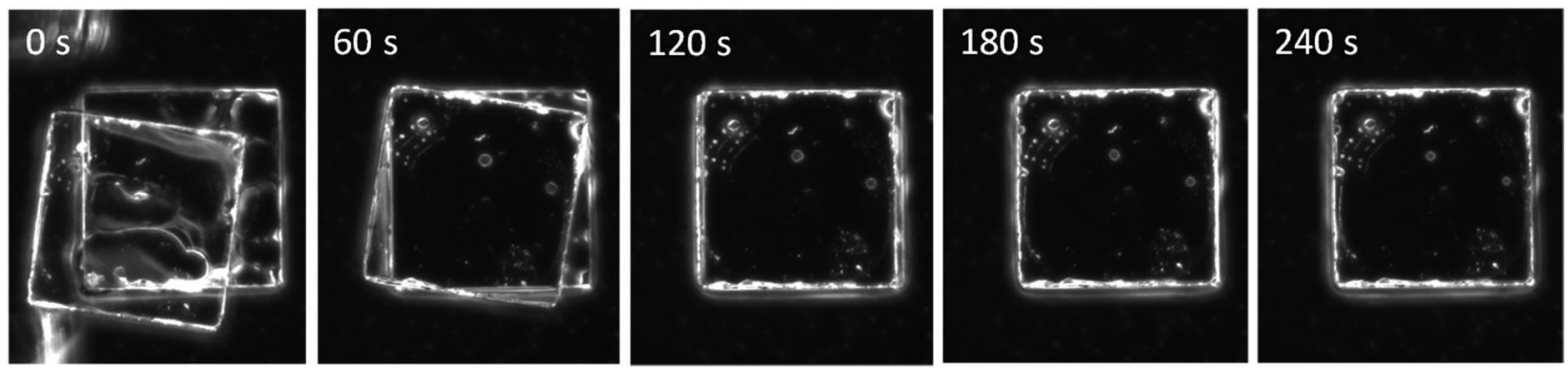

Fig. 12 Snapshots from the recording of the capillary self-alignment of two stacked and shape-matching $300 \mu \mathrm{m} \times 300 \mu \mathrm{m}$ SU- 8 chips mediated by an interposed highly viscous UV adhesive (full video available in ESI†). 
(a)

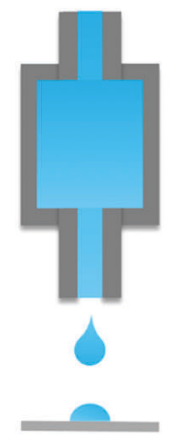

(b)

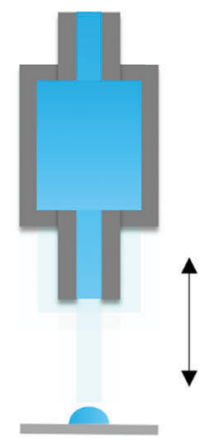

(c)

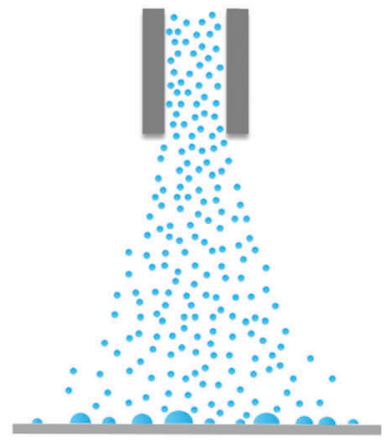

(d)

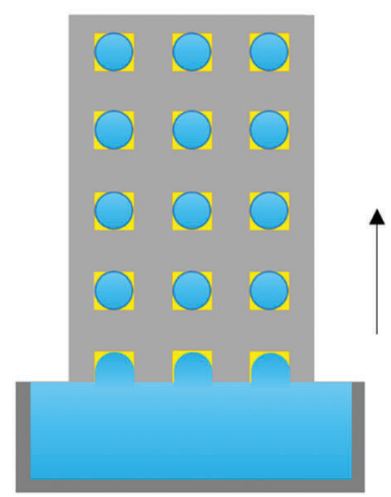

Fig. 13 Common methods of liquid deposition: (a) non-contact, single droplet; (b) contact, single droplet; and (c) parallel stochastic; (d) parallel deterministic.

Liquid deposition methods can be divided into four main categories: (a) non-contact, single-droplet methods; (b) contact, single-droplet methods; (c) parallel stochastic methods and (d) parallel deterministic methods. Fig. 13 illustrate the working principle of the different liquid deposition methods, and Table 2 summarizes their main features.

In non-contact, single-droplet methods a droplet is shot from a nozzle to the receptor, similarly to inkjet printing (Fig. 13a). Numerous receptors can be addressed efficiently and in sequence with the help of a motorized positioning system when the dispenser is shooting at a high speed. The resulting single droplet volume can measure down to tens of picoliters. ${ }^{89,90}$ Such non-contact dispensers are suitable for water-based and low viscosity fluids (e.g. $\left.5 \mathrm{mPa},{ }^{90} 20 \mathrm{mPa}^{89}\right)$. More viscous liquids can also be handled, provided their viscosity is reduced (e.g. by heating) below a dispenser-dependent threshold value. ${ }^{88}$ During operation, the tip of the dispenser is kept a few millimeter away from the receptors. A distance of tens of millimeters is possible with reduced reliability. Acoustic liquid handling ${ }^{94}$ is an alternative technique, in which high-frequency acoustic signals are focused on the surface of a fluid of interest to eject sequentially and directionally droplets of $\mathrm{nL}$ to $\mathrm{pL}$ volumes with high accuracy and precision. ${ }^{95}$

Contact, single-droplet methods (Fig. 13b) include a variety of dispensers and techniques, from traditional time-pressure dispensers and pump dispensers to nano-contact printing. In all these methods, a single droplet is formed when the nozzle of a needle comes into contact with the receptor, followed by liquid injection, pinch-off and removal of the needle. Nanoliter droplets are typically achievable with commercial dispensers. With more precise control of the contact time profile, smaller volumes are also achievable. Similarly to non-contact methods, the addressing of multiple receptors require proper positioning systems. Contact dispensers can dispense a wide range of liquids, from aqueous to very viscous.

Parallel stochastic methods (Fig. 13c) provide an efficient way to deposit at once liquids to a large amount of receptors or component-receptor pairs. In parallel stochastic methods, liquid is delivered randomly to the substrate $e$.g. by condensation from vapor phase or by water mist droplets impacting on the surface. Compared to the single droplet methods, the parallel stochastic methods can produce a huge amount of individual droplets of down to femtoliter volumes, which makes it highly efficient. The parallel stochastic method is most suitable for water disposition, ${ }^{54,92,96}$ even though other liquids can also be handled. As the deposition takes place on the full substrate, droplets on undesired regions of the substrate (e.g., outside the receptors) should be removed by post-processing (e.g., vaporization) after the self-alignment step, unless their existence is compatible with the subsequent processing steps.

Parallel deterministic methods, such as dip-coating (Fig. 13d), can deposit liquid to a large amount of receptors. ${ }^{42,59}$ In dipcoating, the substrate patterned with receptors is dipped into the liquid to be coated and then redrawn from the liquid. ${ }^{44,93}$ Depending on the receptor types, either chemically of topographically defined (see Section 3.1), the coating dynamics and particularly the failure of the liquid bridges can change significantly. ${ }^{44}$ In the case of chemical patterning, the receptors should be hydrophilic, while the substrate should be sufficiently hydrophobic such that only the receptor will be coated and the substrate will be dewetted by the liquid. Compared to e.g. lines, the coating of closed chemically-patterned patches, like the receptors, involves capillary break-up effects that are intrinsically time-dependent. ${ }^{43}$ The thickness of the coated liquid depends on the pulling speed and insertion angle of the substrate, the surface tension, viscosity and density of the liquid, the surface energy of the receptors and the substrate (i.e., the wetting contrast), and the shape, size and orientation of the receptors. ${ }^{42,43,93}$ Coating thickness down to $1 \mu \mathrm{m}$ (equivalent to $\left.1 \mathrm{~nL} \mathrm{~mm}{ }^{-2}\right)^{93}$ and sessile droplets of femtoliter volumes ${ }^{44}$ can be obtained by dip-coating. Additionally, low melting point solders are typically deposited on receptors by this technique. ${ }^{59}$ Selective liquid deposition onto hydrophilic receptors can also be carried out using droplets sliding over hydrophobic substrates. ${ }^{97}$ 
Table 2 Common methods for liquid dispensing

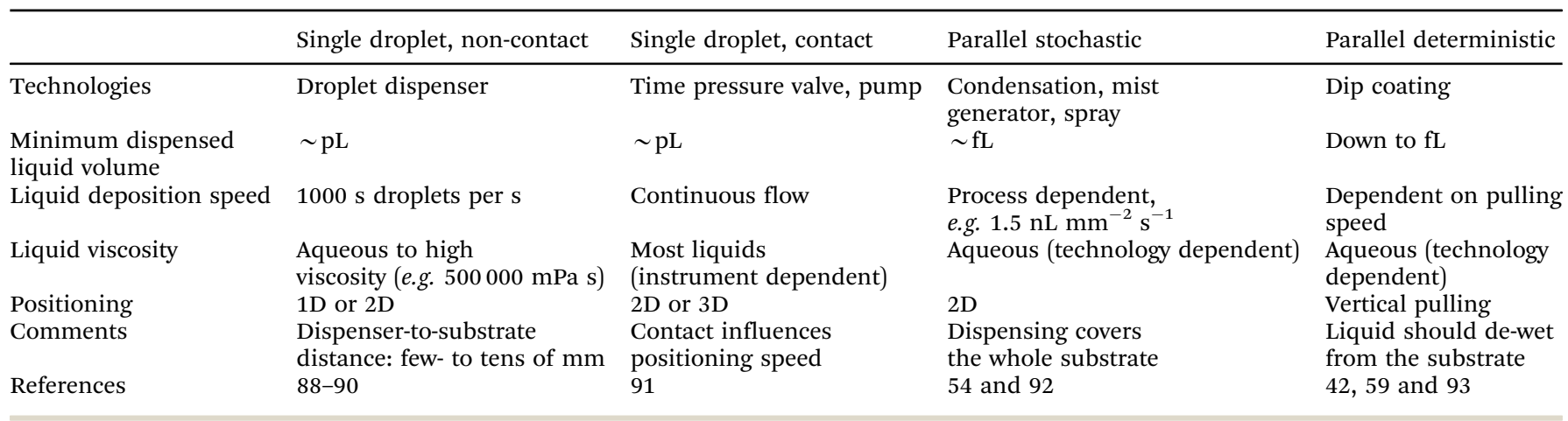

Many parameters guide the liquid choice, including the ability to wet the chosen surfaces, electrical or thermal conductivity, optical transparency, the ability to make permanent bonding through phase change (e.g. by freezing, optically- or thermally-initiated cross-linking), and processing temperatures. The three main types of liquids that have been applied in capillary self-alignment are solders, water and other solvents, and adhesives. Their specific properties are discussed in the following sections.

6.1.2 Solders. Capillary self-alignment mediated by solder droplets in air was the first extensively studied self-alignment process, in the context of flip-chip ${ }^{98-101}$ and surface-mount reflow soldering. ${ }^{102,103}$ The self-centering force of the solder joint was clearly identified in early work ${ }^{103}$ focusing on soldering defects. One of the major applications identified for solder selfalignment was for passively aligned optical interconnects. ${ }^{104}$ The high accuracy requirements of optical interconnects could not be achieved using solder self-alignment alone, but capillary self-alignment was usually combined with mechanical shape matching or stoppers to achieve accuracies better than $1 \mu \mathrm{m},{ }^{100,104}$ suitable for interconnects. Variations in solder volume can cause undesired variations in chip-to-substrate gap, or tilts when multiple solder balls are used. These variations can be avoided by using mechanical spacers. ${ }^{105}$

Molten solders characteristically have high surface tension and low viscosity (Fig. 11), and are therefore well suited for selfalignment. Additionally, solders can make permanent bond upon cooling and serve as an electrical connection (Section 5). Since solder balls are also used as electrical connectors, typically multiple solder joints are used, laid out in a row or a matrix pattern (ball grid arrays). The multipurpose role of solder joints means that solders are usually not chosen solely based on the self-alignment properties. Environmental aspects also limit the choice: lead-based solders are banned or limited in commercial use, e.g. by the Restriction of Hazardous Substances Directive (RoHS) in the EU. Examples of solders used for self-alignment include $\mathrm{Sn} / \mathrm{Pb}\left(5 / 95^{100}\right.$ or eutectic $\left.63 / 37^{105}\right), \mathrm{Au} / \mathrm{Sn}^{106}\left(78 / 22^{107}\right.$ or eutectic 80/20 $\left.{ }^{108,109}\right)$ and $\mathrm{In} / \mathrm{Pb}(50 / 50) .{ }^{110}$

One issue with traditional solders can be the required high melting temperatures, not compatible with heat-sensitive materials and components. Low melting point solders, ${ }^{111,112}$ such as $\mathrm{Bi} / \mathrm{Sn} / \mathrm{Pb}(46 / 34 / 20)^{73}$ are therefore used. Another issue, often hindering solder self-alignment and interconnect reliability, is insufficient wetting or adhesion caused by solder or pad oxidation. This can be solved by using liquid flux during reflow, or by removing the oxide prior to the reflow and selfalignment in protected environments ${ }^{104}$ (see also Section 6.3).

6.1.3 Water and other solvents. As for solvents, water droplets in air have mostly been used for capillary self-alignment. Water is a polar solvent and has a relatively high surface tension, resulting in large forces for self-alignment. Water is an attractive choice also because it is easily available, non-toxic and compatible with many materials and processes. Moreover, (super)hydrophobic surfaces can be fabricated in many ways and can be used as non-wetting areas for self-alignment substrates (Section 3.1).

Water alone does not achieve a permanent bond, even after evaporation. By using silicon oxide surfaces, and adding small fractions of hydrogen fluoride (HF) into the water, components can be chemically and permanently bonded to receptors. ${ }^{113}$ Alternatively, several authors have explored a two-phase process, ${ }^{114-116}$ whereby the initial self-alignment is done using large hydrophilic receptors and commensurate water droplets. Metallic (e.g. Au finished) bonding pads and corresponding solder bumps are contained inside the droplet on receptors and components. After the self-alignment, the bumps are bridged to the contact pads. Adding mild acids to the water droplet prevents solder oxidation during capillary self-alignment. ${ }^{115}$

Other solvents that have been used for self-alignment include ethylene glycol. ${ }^{117}$ Ionic liquids represent moreover an interesting and so far overlooked option. Ionic liquids are salts with low melting points (typically below $100{ }^{\circ} \mathrm{C}$ ) and moderate electrical conductivity. They afford negligible volatility and a wide range of surface tension values depending on their chemical composition. ${ }^{118,119}$

6.1.4 Adhesives. Adhesives have several attractive properties for capillary self-alignment. ${ }^{120}$ Many adhesives are liquid at room temperatures, and only after being heated up they cure to make a permanent bond. The downside is that many such adhesives have small surface tension, and are not easily confined inside receptors (Section 3.1). The problem can be solved by increasing the interfacial tension, for instance by immersing the adhesive droplet in an immiscible liquid. The interfacial tension of all hydrocarbons (such as hexadecane, ${ }^{27}$ besides adhesives) in contact with water increases ${ }^{121}$ so that 
they can be confined within hydrophobic areas. Electrical connections in adhesive-based capillary self-alignment can also be established by using anisotropic conductive adhesives (ACAs), ${ }^{48,122}$ together with low-melting point alloys. ${ }^{122}$

\subsection{Component feeding}

Before capillary self-alignment can take place, the components need to be brought into contact with the liquid on the receptors. Three main strategies have been devised for component feeding: deterministic feeding, in series or in parallel, and parallel stochastic feeding.

For deterministic feeding, the components can be fed using a robotic pick-and-place device, either in series ${ }^{17,117,123}$ or in parallel. ${ }^{18,72,124}$ Parallel deterministic feeding includes laserinduced release and transfer from a donor substrate, ${ }^{125,126}$ rollto-roll mechanisms, ${ }^{12,127}$ parallel component handling over temporary carriers ${ }^{92,124}$ and floatation. ${ }^{59,76}$ Feeding can be accomplished also stochastically, as often done in fluidic selfassembly. ${ }^{28,29,61,111,128,129}$ In this case, the components are introduced to a liquid bath and driven towards the receptors using fluidic flow and/or vibration to impose movement and overcome friction and component aggregation. The main feeding strategies are illustrated in Fig. 14 and their properties summarized in Table 3 .

Single component pick-and-place is a widely used process in precision industrial manufacturing, whereby a robot moves a gripper to pick components from a dicing tape or other carriers and bring them to the receptors (Fig. 14a). Besides vacuum grippers, other tools such as mechanical tweezers and capillary grippers are used..$^{60,75,130,131}$ The performance of single component pick-and-place can reach tens of assembled components per second, with an accuracy of e.g. $10 \mu \mathrm{m}$. For example, for a typical linear chip size of $1 \mathrm{~mm}$, a high-end assembly system like the FCM 10000 (Mühlbauer AG, Roding, Germany) is able to assemble $\sim 8000$ chips per hour with a placement accuracy of $30 \mu \mathrm{m} .{ }^{132}$ The accuracy can be significantly improved only by reducing the operating speed. Capillary self-alignment provides a simple and remarkable way to overcome such throughput/ accuracy trade-off: it is an attractive method to let pick-andplace reach the highest accuracy without sacrificing speed.

Parallel pick-and-place using multiple vacuum or capillary grippers is an interesting batch feeding process (Fig. 14b). A closely related technology is wafer-level transfer of the components on an intermediate carrier before the components are placed onto the final target. ${ }^{124}$ The challenge is to meet simultaneously the operational conditions, particularly the alignment and orientation, for all components in the batch during the pick-and-place, especially when the number of components is as high as in typical batch fabrication. Therefore, the parallel pick-and-place process requires careful engineering and high-speed operation.

Laser-induced release of components ${ }^{125,126,135,136}$ can directly operate on the donor substrate, such as a transparent wafer or dicing tape, and combines the component release step directly with feeding to receptors (Fig. 14c). This is in contrast with all other methods, where the chip should be transferred to intermediate media such as gripper, transfer wafer, feeding container or liquid surface. The limitation of this methods lies in the possible reorientation of the component upon the abrupt detachment process. ${ }^{136}$

Fluidic feeding and vibrational agitation is a widely used method for fluidic self-assembly (Fig. 14d). The structure hosting the assembly system can be a bath, ${ }^{28,29,137}$ a rotating drum, ${ }^{78}$ or a reel-to-reel machine. ${ }^{127}$ The benefit lies in the parallelism and precision of the process achieved without high-precision mechanism. The drawback is the randomness of the assembly process, which (a)
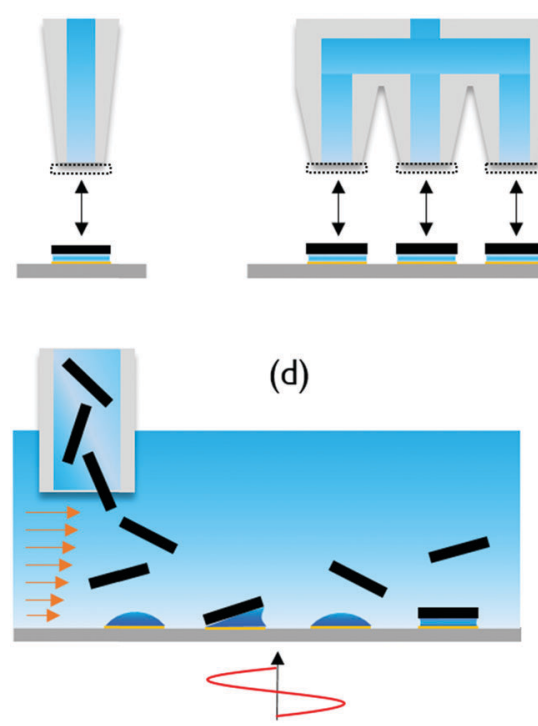

(b)
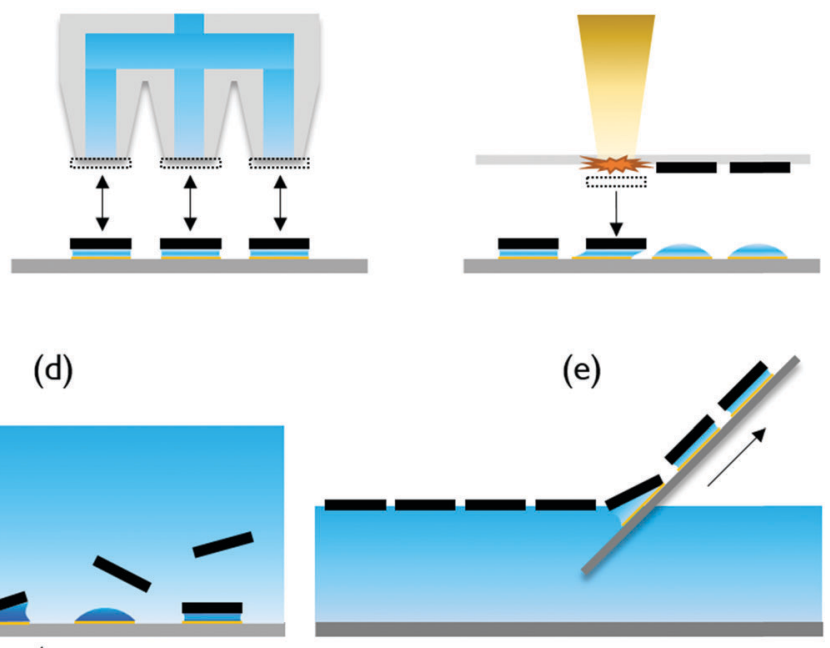

(c)

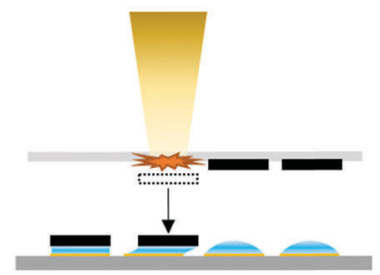

(e) 
Table 3 Comparison of component feeding techniques

\begin{tabular}{|c|c|c|c|c|c|}
\hline Method & Single component pick-and-place & Parallel pick-and-place & $\begin{array}{l}\text { Laser-induced } \\
\text { releasing }\end{array}$ & $\begin{array}{l}\text { Fluidic feeding } \\
\text { and vibration }\end{array}$ & Floatation feeding \\
\hline Speed [unit per s] & High-speed: $>15$ low-speed: $<1$ & Process dependent & Several & Few to 500 & $>1000$ \\
\hline Comments & Speed reduces accuracy & $\begin{array}{l}\text { Careful alignment } \\
\text { for high parallelism }\end{array}$ & $\begin{array}{l}\text { Directly using } \\
\text { the dicing tape }\end{array}$ & $\begin{array}{l}\text { Non-deterministic } \\
\text { feeding }\end{array}$ & $\begin{array}{l}\text { Chip should float } \\
\text { on/in the liquid }\end{array}$ \\
\hline References & 19, 60 and 133 & 124 & 134 & $12,13,28$ and 29 & 59 and 77 \\
\hline
\end{tabular}

leads to non-deterministic results and complicates the construction of sophisticated structures. Moreover, the fluidic self-assembly process differs significantly from state-of-the-art semiconductor back-end-of-line processes. Nevertheless, randomness can be partly compensated by high components-toreceptors ratios, and highly efficient systems can be designed after careful engineering. ${ }^{127}$

The feeding mechanism exploiting component floatation has demonstrated the highest assembly rate to date ${ }^{59}$ (Fig. 14e). The process is largely deterministic and resembles flow coating by iterative substrate pulling. The process is facilitated by a prior orientation of the components floating at the immiscible liquid interface determined by the minimization of surface energies (Fig. 16c). The liquid phase not only works as the self-alignment medium but should also be compatible with the other chemical or physical properties of the components (e.g. energy, density), and a mechanism to release the component into the liquid.

Mechanical trapping based on three-dimensional geometric features has been exploited to direct component assembly, program multistep assembly sequences and enforce unique component orientation. Examples include stochastic component feeding over a planar template for batch pre-alignment, ${ }^{92}$ alignment wells and pedestals for univocal component assembly, ${ }^{78}$ shape-specific recessed receptors for the simultaneous and non-interferring assembly of matching components ${ }^{128,137}$ and three-dimensional shape-encoded sequential self-assembly. ${ }^{138}$

Capillary self-alignment can correct the initial translational and rotational offsets of the feeding step relative to the final, correctly aligned position (see Section 2.2). A few studies analysed the influence of the component feeding offset on the success of capillary self-alignment. ${ }^{26,53,72,120}$ Sariola et al. experimentally investigated the alignment yield upon varying translational biases of sub-millimeter square components. ${ }^{60}$ The main result was that the yield dropped much faster with normal bias than with lateral bias, because lateral bias only shears the meniscus, while normal bias can result in either bulging of the liquid from the sides or necking of the meniscus (Fig. 15). Capillary self-alignment through biases as large as half the sidelength of the components were demonstrated in the assembly of silicon dies ${ }^{116}$ and plastic devices. ${ }^{48}$ Such initial displacements are associated with an initial dewetting of the bottom surface of the component by the meniscus, as captured by a recent piece-wise model. ${ }^{33}$ In the model, an extension of the one shown in Section 2.3, the lateral restoring force depends on the liquid contact angle upon unpinning of the contact line, besides on the geometry of the meniscus, and it is predicted to saturate to a constant limit value for offsets larger than the gap.

The maximum offset that can lead to successful capillary self-alignment can actually be larger than the size of the receptor. Particularly, self-transport and self-alignment of microcomponents was reported, whereby even a gap of tens of microns between a component and its shape-matching receptor, defined chemically on a superhydrophobic substrate, is bridged by means of microscopic rain. ${ }^{96}$

\subsection{Post-processing}

Successful capillary self-alignment leads to the registration of the components to the underlying receptors. In some manufacturing applications, eminently in electronic assembly and

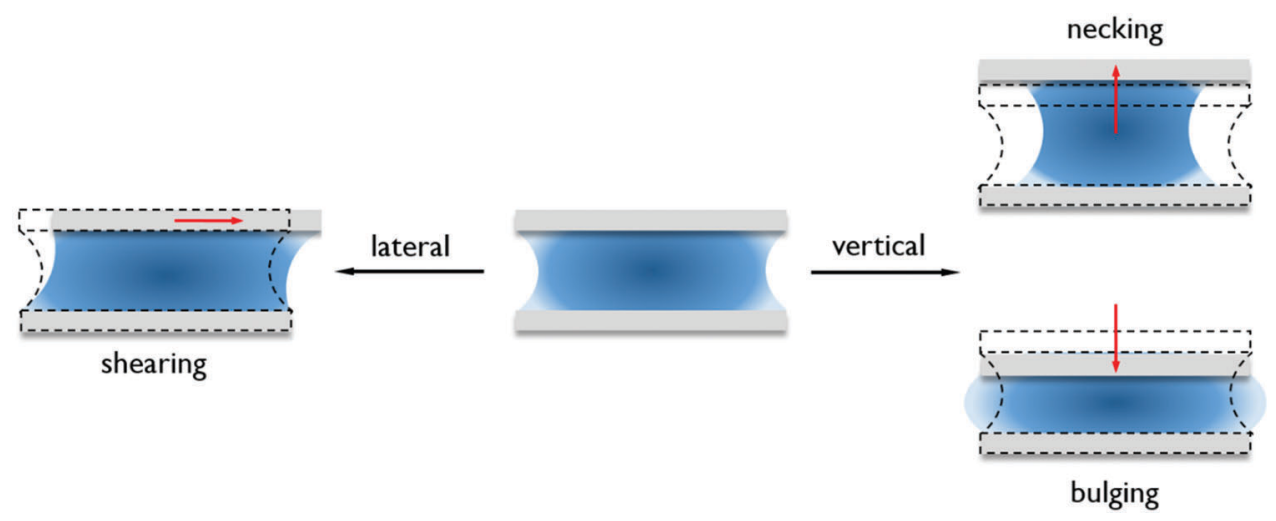

Fig. 15 Lateral versus normal component displacement with respect to the equilibrium configuration (sketched in wireframe). Lateral displacement (left) only shears the fluid joint. Normal displacement (right) can cause necking (top) or bulging (bottom). 
packaging, the finalization of the assembly process further requires the establishment of a stable connection between components and receptors to form a functional system. In this case, capillary self-assembly becomes an important step within a more comprehensive heterogeneous integration process, and compatibility needs to be assured. ${ }^{21}$

The component-to-receptor connection can be simply mechanical or additionally electrical. In the former case, the component only needs to be attached to the receptor. Volatile liquids, such as water-based solutions, rapidly evacuate the gap after capillary self-alignment leaving the surfaces of component and receptor in direct contact. This can lead to capillary adhesion, which was shown to be viable for temporary transfer of components to carriers, ${ }^{124}$ or even to covalent bonding mediated by chemical reactions, for instance by using diluted hydrogen fluoride between oxide-passivated surfaces. ${ }^{113}$ Alternatively, glues or epoxies can be used as lubricants, which can be optically or thermally cross-linked to provide the mechanical connection. $^{29}$

A few strategies can be used to electrically beside mechanically interconnect components to receptors. The earliest and simplest strategy consists in the use of an electrically conductive fluid material as the medium for the self-alignment. Dating back to the original IBM's C4 process, ${ }^{14}$ molten solder driven self-alignment ${ }^{111,112}$ has been object of intense research through the years and is still actively employed in advanced implementations of parallel roll-to-roll stochastic assembly of microcomponents ${ }^{12,59,78,127}$ (see also Sections 3.1.1 and 6.2.1). In this version of capillary self-alignment, a solder material is coated on the metal-finished surface of the receptor or of its fractions where the interconnections need to be established. The solder can be applied in several ways, e.g., by dip-coating, ${ }^{27,111,127}$ screen printing, or electroplating. ${ }^{21,29,115}$ The high surface tension of the molten solder, wetting both receptor and component, promotes the final self-alignment. However, this requires that the solder material be kept in the molten state and free of superficial oxide throughout the process until final solidification. When in air, molten-solder self-alignment is akin to flipchip assembly and is performed under reducing atmospheres, such as formic acid, that continuously reduce the solder surface. When performed by fluidic means, the process is hosted in warm acidic solutions. ${ }^{111,127}$ It should be noted that the protracted growth and removal of oxide erodes the material and can severely damage the interconnections. ${ }^{111,112}$ This issue significantly affects the yield of assembly processes that use small-sized interconnections such as microbumps. In contrast to original demonstrations and proofs of concept, lead-free solder alloys with relatively low melting points, such as bismuth- and indiumbased, are by now widely used (Section 6.1.2).

Alternatively, a two-step approach can be exploited, whereby the electrical interconnections are formed after the finalization of the capillary self-alignment. For instance, Xiong et al. used an epoxy as liquid meniscus for self-alignment, and copper electroplating to bridge the receptors to the components after their placement. ${ }^{29}$ More commonly, after capillary self-alignment employing an acidic solution for the meniscus, a thermal treatment under vertical loading is applied at substrate level to establish in parallel the interconnections between matching arrays of metal pads and corresponding oxide-free solder bumps at single component level. Such thermo-compression bonding makes use of solder-specific time-programmed thermal reflow profiles to ensure the formation of high-quality and reliable metal bonds (i.e., showing no voids, good adhesion, and electrical contacts with low resistance and low parasitic components). ${ }^{18,116}$ Thermo-compression bonding mediated by anisotropic conductive adhesives (ACAs) was successfully demonstrated in a capillary self-alignment process. ${ }^{48}$ Though featuring higher electrical resistances compared to fully metallic solders, ACAs offer a distinct dispensing advantage: they can be coated over arrays of interconnects of arbitrary spatial distribution without inducing electrical short-circuits or cross-talks, thanks to their anisotropic and pressure-dependent electrical conductivity. ${ }^{122}$ Recently, advanced approaches that skip the use of solder microbumps in favour of direct metal insertion ${ }^{139}$ and metal/dielectric bonding, such as e.g. copper/copper ${ }^{140}$ and copper/oxide, ${ }^{141}$ were also successfully demonstrated.

As earlier mentioned, a further alternative for known good components is to be first ordered by capillary self-alignment over a temporary carrier, in this case with their bonding side facing up. The carrier is then flipped and aligned onto the target substrate, to which the components are contextually bonded and released. ${ }^{18,124}$ This approach is suitable for sequential threedimensional stacking of components onto target substrates, ${ }^{18}$ yet it suffers from the combined registration tolerances of the capillary self-alignment and wafer-level alignment steps.

\subsection{Specific implementations}

6.4.1 High throughput. High-throughput precision assembly is one of the main advantages of capillary self-alignment. Both robotic assisted hybrid assembly and fluidic self-assembly have shown promising results.

The combination of high-speed robotic feeding with capillary self-alignment has been tested in semiconductor industrial settings. Capillary self-alignment has been integrated with highspeed die pick-and-place machines, using receptors pre-coated with adhesive ${ }^{142}$ (Fig. 16a). The pick-and-place robot alone normally operated at a throughput of over 40000 units per hour (UPH, i.e., >15 unit per s) with an accuracy of about $10 \mu \mathrm{m}$ for $300 \mu \mathrm{m}$ silicon components on lead-frames. Assisted by capillary self-alignment, the assembly accuracy reached $1 \mu \mathrm{m}$ with respect to the receptors and $2 \mu \mathrm{m}$ in the global coordinate system, without sacrificing the throughput. Assembly yield reached $98 \%$ in the tests.

An automated roll-to-roll fluidic self-assembly setup has been recently developed, which combines stochastic fluidic component feeding, substrate vibration, a rolling mechanism for the movement of the flexible substrate, and component recycling (Fig. 16b). Self-assembly of 300 to $1000 \mu \mathrm{m}$-sized square silicon components onto $2.5 \mathrm{~cm}$-wide polyimide-based web was demonstrated using low melting point solder as self-aligning medium in a mildly acidic, water-based solution at $80{ }^{\circ} \mathrm{C} .{ }^{12,127} \mathrm{~A}$ throughput of $15000 \mathrm{UPH}$ ( $>4$ unit per s) and a yield larger than 99\% has been reported, without indicating the actual assembly accuracy. 

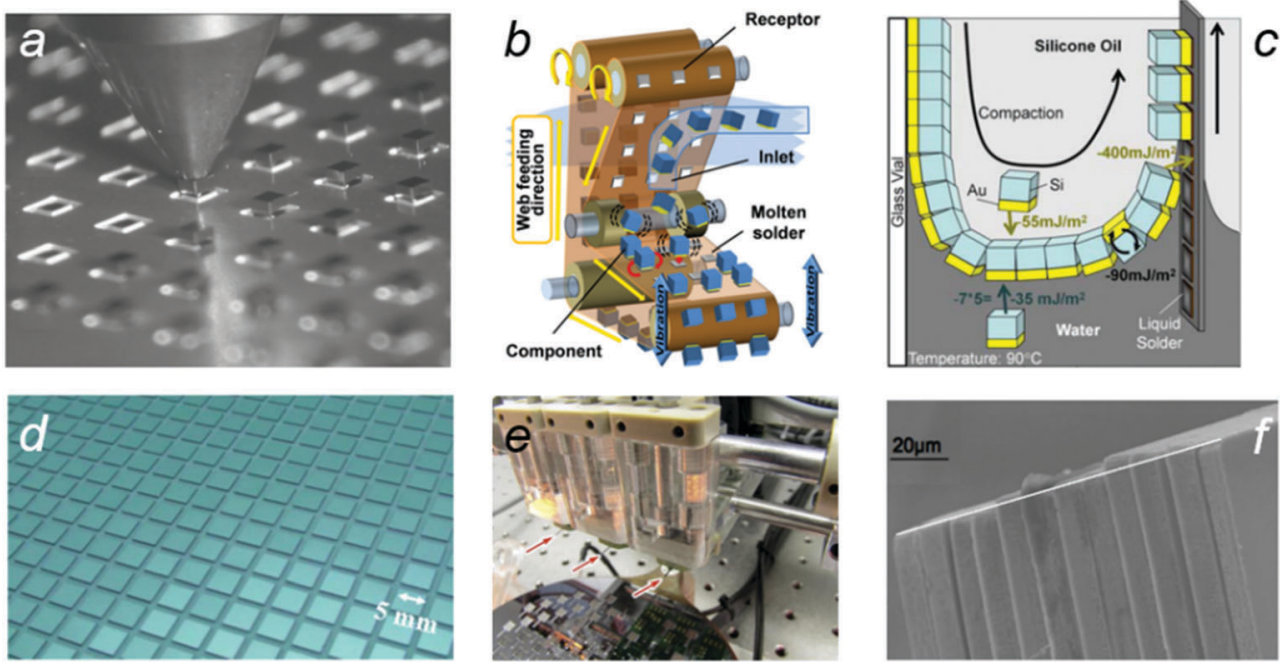

Fig. 16 Applications of capillary self-alignment. (a) Industrial high-throughput robot using adhesive self-alignment to assemble $300 \mu$ m-sized microchips on leadframe; ${ }^{142}$ (b) automated roll-to-roll fluidic self-assembly machine to assemble 300 to $1000 \mu \mathrm{m}$-sized square silicon components onto $2.5 \mathrm{~cm}$-wide polyimide-based web using low melting point solder (reprinted with permission from ref. 12, (C) 2014, John Wiley \& Sons); (c) 20-60 $\mu$ m-sized semiconductor components assembled on a vertically pulling substrate at the silicon oil/water/substrate triple interface with a batch assembly throughput of 62500 chips in 45 seconds (reprinted with permission from ref. 59, (C) 2010 National Academy of Sciences); (d) assembly of arrays of 20 mm dies on carrier substrates through a combination of parallel vacuum gripping and capillary self-alignment (reprinted with permission from ref. 113, (C) 2010, American Institute of Physics); (e) millimeter sized MEMS force sensors picked up and aligned to a PCB by the capillary force of molten solders between matching pads on both the PCB and the sensors (reprinted from ref. 143); (f) sequential stacking of $10 \mu \mathrm{m}$-thick silicon dies using robotic pick-and-place and capillary self-alignment (reprinted with permission from ref. 79, (C) 2013 IEEE).

The intrinsic parallelism of fluidic self-assembly makes the process scalable to the integration of microcomponents onto arbitrarily large substrates, suitable for macroelectronic applications such as flexible substrates for solid-state lighting.

6.4.2 High precision. Capillary self-alignment can easily achieve sub-micron registration accuracy. ${ }^{28,59-61,63,74}$ Whereas the highest absolute accuracy is normally achieved for submillimeter components, millimetre-sized components have also achieved similarly high relative accuracy.

In ref. 113, droplets of diluted hydrofluoric acid have been used to align arrays of $5 \times 5 \mathrm{~mm}^{2}$ chips onto hydrophilic receptors on a substrate (Fig. 16d). Average and best alignment accuracy were $400 \mathrm{~nm}$ and $50 \mathrm{~nm}$, respectively. Dubey et al. have recently demonstrated accurate $(<2 \mu \mathrm{m})$, fast and good dielectric bonding of $10 \times 10 \mathrm{~mm}^{2} \mathrm{Si}$ dies for 3D integration applications through a capillary self-alignment process. $^{84}$ They reported a systematic improvement in alignment accuracy to substrate receptors when rapid substrate heating was enforced as compared to room temperature and slow heating bonding conditions, and tentatively attributed the result to surface tension gradients and reduced viscous resistance (see Section 5) induced by the thermal treatment.

The precision of capillary self-alignment is also rather robust against jaggedness and poor definition of receptor edges. As seen in Section 4.2, the achieved accuracy can be better than the mean profile deviation from the smooth edge shape.

6.4.3 Component flipping. In addition to achieving lateral component movement and component rotation around the vertical axis, the surface tension of a droplet can be used to power rotations around in-plane axes. This principle was engineered to deploy three-dimensional MEMS devices, ${ }^{15}$ to

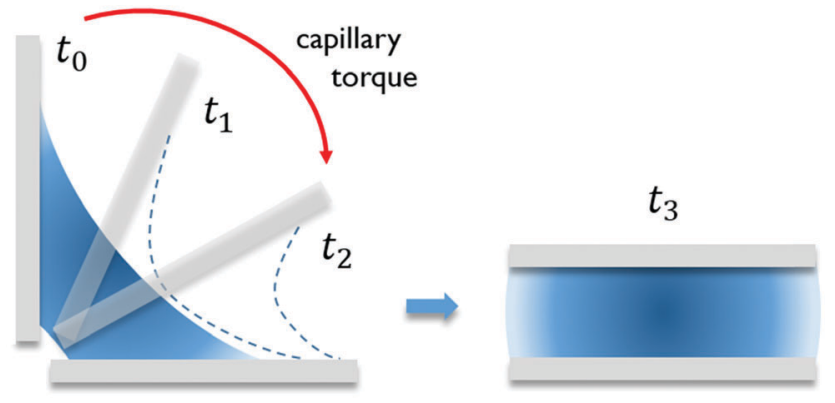

Fig. 17 Using surface tension of a droplet to flip components. The top component is wetted by the liquid meniscus and upon release is free to move under the action of the capillary torque. The component consequently rotates until its wet side faces the receptor.

self-fold patterned micro- and nanoparticles, ${ }^{16}$ and to actuate three-dimensional voxels. ${ }^{4}$ Capillary forces are also congenial to efficiently flip parts in place (Fig. 17). For instance, $100 \times$ $50 \mu \mathrm{m}$ microchips were flipped and temporarily bonded to the target structure by robotic manipulation and capillary selfalignment - an operation that would otherwise require either dexterous manipulations, complicated kinematics capable of object-centric rotations, or rotating the part by pushing it against an external object. ${ }^{144}$

\section{Patents}

Patents provide an interesting outlook on both potential and commercial use of surface tension-driven self-alignment. 
Patents can at times disclose works that are not redundant with or that are prior to academic research published in journals. Relevant patents with these characteristics are mentioned here below. The search keywords were "surface tension”, "capillary forces”, "assembly”, “packaging”, "SMD components”, “pickand-place", and "grippers".

Patents related to self-alignment root back at least to IBM's 1973 patent, ${ }^{145}$ where we read: "[...] The chip structure permits a method of automatic alignment [...]. The structure is heated to melt the solder mounds, wetting said land pads and thereby moving the chip [...]'. Many companies later patented several additional ideas related to self-alignment: Western Digital Corp. ${ }^{146}$ ("The method includes coating the pad with solder, placing the leads of the components on the solder coated pads, melting the solder and vibrating the leads relative to the pad while the solder is molten, so that the surface tension of the molten solder centers the leads on the pads. The molten solder is then cooled until it resolidifies."), Plesseys Overseas, ${ }^{147}$ Ricoh, ${ }^{148}$ Motorola. ${ }^{149}$

Several aspects of fluidic self-assembly have been patented, including a method for self-assembling several, differing components simultaneously into a single system $;{ }^{150}$ a method to assemble microdevices into receptors with shape-complementary geometry; ${ }^{151}$ a method to form self-assembled electrical networks; ${ }^{152}$ methods and apparatuses relating to block configurations, ${ }^{153}$ an apparatus for forming an electronic assembly ${ }^{154}$ and other similar patents from Alien Technology; ${ }^{155-161}$ methods for assembling microcomponents to binding sites ${ }^{162}$ and fabricating stacked chips using fluidic templated assembly; ${ }^{163}$ a system including self-assembled interconnections; ${ }^{164}$ a method for fabricating self-assembling microstructures ${ }^{165}$ and fluidic self-assembly of active antenna; ${ }^{166}$ and a method for mounting anisotropicallyshaped members. ${ }^{167}$ All mentioned patents concern fluidic selfassembly whereby the components are delivered to the receptors through a fluidic suspension.

Aspects of flip chip mounting and bumping have been patented, including a mounting and bump forming method ${ }^{168}$ and properly shaped self-aligning micro-bump structures. ${ }^{169}$ In these patents, the self-alignment takes place at solder bump level.

More recently, a new wave of patents were published in Japan (M. Koyanagi, "Method and apparatus for manufacturing integrated circuit device using self-organizing function"; ${ }^{170}$ Tokyo Electron LTD, "Method and apparatus for producing 3D integrated circuit",171), France (CEA, "Self-Assembly of Chips on a Substrate"172) and Germany (Mühlbauer AG, "Method and apparatus for manufacturing an electronic assembly", ${ }^{173}$ wherein "A chip [...] as an electronic component, is deposited on a carrier substrate $[.$. . by free fall motion [...] by contacting the liquid meniscus of the deposited liquid. Because of the minimization of the free interfacial energy, the chip is aligned on the flexible substrate [...]. As a variant, the liquid can be a heat-curable adhesive with low viscosity").

The patents highlight an evolution from large components, put in contact with many solder bumps, to hybrid assembly, in which the component is dropped in free fall in the vicinity of the target, to the more recent introduction of non-solder liquids, such as curable adhesives with low viscosity.

\section{Outlook}

In this review we have presented the current knowledge and state-of-the-art of surface tension-driven self-alignment. Despite of its apparent simplicity, it was shown that the process is controlled by many physical, material and technological parameters which can significantly affect its throughput and yield. The considerable potential of capillary self-alignment was illustrated through several recent experimental applications. By the combination of robotic component feeding with passive accurate component positioning, capillary self-alignment makes assembly process flows more effective, as it can overcome the current trade-off between assembly throughput and component placement accuracy. Such comparative advantages prompt the technique to be progressively adopted in precision industrial manufacturing, whereby electronic packaging and advanced assembly applications can be particularly benefited.

Most research has focused so far on the assembly of inorganic components. Nonetheless, surface tension can well be engineered for precise handling and assembly of organic materials: many of such materials do express their functionality in presence of liquids, and in turn physiological liquids have physical properties (e.g., surface tension, viscosity, density) well compatible with or suitable for capillary self-alignment. Furthermore, assembly of thin, fragile and flexible components is already being tackled through the technique, as well as self-alignment on soft substrates. ${ }^{174}$

In contrast to its statics, the dynamics of capillary selfalignment is not yet fully captured within a theoretical model. Besides open theoretical questions, further challenges for surface tension-driven self-alignment include mastering the handling of small liquid volumes: how to controllably and reliably deliver very small amounts of liquids, and how to engineer reliable surface tension applications despite intrinsic variations of liquid volume due to evaporation, spreading and contact angle hysteresis. Open impactful opportunities can conversely be envisaged through the control of surface tension effects by external stimuli, such as temperature, surfactants, light and electric fields.

\section{Acknowledgements}

This work was partially supported by the Academy of Finland (project no. 268686, IKARUGA, 2013-2016, project no. 295006, CyberCellulose 2016-2017), and through years by ULB, CNRS, IAP 7/38 MicroMAST funded by BELSPO, and the FNRS.

\section{References}

1 D. Bonn, J. Eggers, J. Indekeu, J. Meunier and E. Rolley, Rev. Mod. Phys., 2009, 81, 739-805.

2 P. Lambert, Surface tension in microsystems, Springer, Berlin, 2013. 
3 S. Herminghaus, Wet granular matter, World Scientific, Singapore, 2013.

4 M. Mastrangeli, Adv. Mater., 2015, 27, 4254-4272.

5 R. Hensel, C. Neinhuis and C. Werner, Chem. Soc. Rev., 2016, 45, 323-341.

6 P. Lambert, Capillary forces in microassembly, Springer, Berlin, 2007.

7 N. Vogel, M. Retsch, C.-A. Fustin, A. del Campo and U. Jonas, Chem. Rev., 2015, 115, 6265-6311.

$8 \mathrm{~J}$. Berthier and K. Brakke, The physics of microdroplets, Wiley, 2012.

9 M. Mastrangeli, S. Abbasi, C. Varel, C. van Hoof, J.-P. Celis and K. F. Böhringer, J. Micromech. Microeng., 2009, 19, 083001.

10 M. Gauthier and S. Régnier, Robotic Micro-Assembly, John Wiley \& Sons, 2011.

11 J. Cecil, M. B. Bharathi Raj Kumar, Y. Lu and V. Basallali, Int. J. Adv. Manuf. Technol., 2015, 83, 1569-1581.

12 S. C. Park, J. Fang, S. Biswas, M. Mozafari, T. Stauden and H. O. Jacobs, Adv. Mater., 2014, 26, 5942-5949.

13 C. J. Morris, S. Member, S. A. Stauth and B. A. Parviz, IEEE Trans. Adv. Packag., 2005, 28, 600-611.

14 L. F. Miller, IBM J. Res. Dev., 1969, 13, 239-250.

15 R. R. A. Syms, E. M. Yeatman, V. M. Bright and G. M. Whitesides, J. Microelectromech. Syst., 2003, 12, 387-417.

16 J.-H. Cho, M. D. Keung, N. Verellen, L. Lagae, V. V. Moshchalkov, P. Van Dorpe and D. H. Gracias, Small, 2011, 7, 1943-1948.

17 V. Sariola, Q. Zhou and H. N. Koivo, Journal of Micro-Nano Mechatronics, 2008, 4, 5-16.

18 T. Fukushima, J. Bea, H. Kino, C. Nagai, M. Murugesan, H. Hashiguchi, K.-W. Lee, T. Tanaka and M. Koyanagi, IEEE Trans. Electron Devices, 2014, 61, 533-539.

19 Q. Zhou, V. Liimatainen and I. Routa, Proceedings of the 5th Electronics System-integration Technology Conference (ESTC), 2014, 1-6.

20 G. Arutinov, E. C. P. Smits, P. Albert, P. Lambert and M. Mastrangeli, Langmuir, 2014, 30, 13092-13102.

21 M. Mastrangeli, W. Ruythooren, J.-P. Celis and C. Van Hoof, IEEE Trans. Compon., Packag., Manuf. Technol., 2011, 1, 133-149.

22 A. W. Adamson and A. P. Gast, Physical chemistry of surfaces, Wiley, 1997.

23 J. B. Bostwick and P. H. Steen, Annu. Rev. Fluid Mech., 2015, 47, 539-568.

24 S.-J. Hong, T.-H. Chou, S. H. Chan, Y.-J. Sheng and H.-K. Tsao, Langmuir, 2012, 28, 5606-5613.

25 J. H. Snoeijer and B. Andreotti, Annu. Rev. Fluid Mech., 2013, 45, 269-292.

26 S. Mermoz, L. Sanchez, L. D. Cioccio, J. Berthier, E. Deloffre and C. Fretigny, 3D Systems Integration Conference (3DIC), 2011 IEEE International, 2012, pp. 1-5, DOI: 10.1109/3DIC.2012.6262953.

27 K. L. Scott, T. Hirano, H. Yang, H. Singh, R. T. Howe and A. M. Niknejad, J. Microelectromech. Syst., 2004, 13, 300-309.

28 U. Srinivasan, D. Liepmann and R. T. Howe, J. Microelectromech. Syst., 2001, 10, 17-24.
29 X. Xiong, Y. Hanein, J. Fang, Y. Wang, W. Wang, D. T. Schwartz and K. F. Böhringer, J. Microelectromech. Syst., 2003, 12, 117-127.

30 P. Lambert, M. Mastrangeli, J. B. Valsamis and G. Degrez, Microfluid. Nanofluid., 2010, 9, 797-807.

31 M. Mastrangeli, J. B. Valsamis, C. V. Hoof, J. P. Celis and P. Lambert, J. Micromech. Microeng., 2010, 20, 075041.

32 J. Berthier, K. Brakke, F. Grossi, L. Sanchez and L. Di Cioccio, J. Appl. Phys., 2010, 108, 054905.

33 M. Mastrangeli, G. Arutinov, E. C. P. Smits and P. Lambert, Microfluid. Nanofluid., 2015, 18, 695-708.

34 J. Berthier, S. Mermoz, K. Brakke, L. Sanchez, C. Frétigny and L. D. Cioccio, Microfluid. Nanofluid., 2013, 14, 845-858.

35 S. Gao and Y. Zhou, Microelectron. Reliab., 2013, 53, 1137-1148.

36 W. Lin, S. K. Patra and Y. C. Lee, IEEE Trans. Compon., Packag., Manuf. Technol., Part B, 1995, 18, 543-551.

37 A. Marchand, S. Das, J. H. Snoeijer and B. Andreotti, Phys. Rev. Lett., 2012, 108, 1-5.

38 P.-S. d. Laplace, Mécanique Céleste: Supplément au livre X, Courcier, Paris, 1805, pp. 349-498.

39 B. Chang, A. Shah, I. Routa, H. Lipsanen and Q. Zhou, Appl. Phys. Lett., 2012, 101, 114105.

40 P. Lambert, A. Chau, A. Delchambre and S. Régnier, Langmuir, 2008, 24, 3157.

41 J. Lu, H. Kuwabara, Y. Kurashima, L. Zhang and H. Takagi, Microelectron. Eng., 2015, 145, 75-81.

42 M. Mastrangeli, W. Ruythooren, C. Van Hoof and J.-P. Celis, J. Micromech. Microeng., 2009, 19, 045015.

43 B. J. Brasjen, A. W. van Cuijk and A. A. Darhuber, Chem. Eng. Process., 2011, 50, 565-568.

44 Y. Wang and T. J. McCarthy, Langmuir, 2014, 30, 2419-2428.

45 B. Chang, V. Sariola, S. Aura, R. H. A. Ras, M. Klonner, H. Lipsanen and Q. Zhou, Appl. Phys. Lett., 2011, 99, 034104.

46 C. G. Tsai, C. M. Hsieh and J. A. Yeh, Sens. Actuators, A, 2007, 139, 343-349.

47 J. F. Oliver, C. Huh and S. G. Mason, J. Colloid Interface Sci., 1977, 59, 568-581.

48 G. Arutinov, M. Mastrangeli, E. C. P. Smits, G. V. Heck, J. M. J. D. Toonder and A. Dietzel, J. Microelectromech. Syst., 2015, 24, 126-133.

49 V. Liimatainen, V. Sariola and Q. Zhou, Adv. Mater., 2013, 25, 2275-2278.

50 T. L. Liu and C.-J. C. J. Kim, Science, 2014, 346, 1096-1100.

51 B. Chang, A. Shah, I. Routa, H. Lipsanen and Q. Zhou, Journal of Micro-Bio Robotics, 2014, 9, 1-10.

52 V. Liimatainen, A. Shah, L. S. Johansson, N. Houbenov and Q. Zhou, Small, 2016, 12, 1847-1853.

53 Y. Ito, T. Fukushima, H. Kino, K. W. Lee, T. Tanaka and M. Koyanagi, J. Microelectromech. Syst., 2016, 25, 91-100.

54 B. Chang, V. Sariola, M. Jääskeläinen and Q. Zhou, J. Micromech. Microeng., 2011, 21, 015016.

55 G. Arutinov, PhD thesis, Eindhoven University of Technology, 2014.

56 V. Liimatainen, V. Sariola and Q. Zhou, The 8th Annual IEEE International Conference on Nano/Micro Engineered and Molecular Systems, 2013, vol. 1, pp. 1088-1091. 
57 J. Berthier, K. Brakke, S. Mermoz, L. Sanchez, C. Fretigny and L. Di Cioccio, Sens. Transducers J., 2011, 13, 44-52.

58 G. Arutinov, M. Mastrangeli, E. C. P. Smits, H. F. M. Schoo, J. Brugger and A. Dietzel, Appl. Phys. Lett., 2013, 102, 144101.

59 R. J. Knuesel and H. O. Jacobs, Proc. Natl. Acad. Sci. U. S. A., 2010, 107, 993-998.

60 V. Sariola, M. Jääskeläinen, Q. Zhou and M. Jääskeläinen, IEEE Trans. Robotics, 2010, 26, 965-977.

61 U. Srinivasan, M. a. Helmbrecht, C. Rembe, R. S. Muller and R. T. Howe, IEEE J. Sel. Top. Quantum Electron., 2002, 8, 4-11.

62 B. Chang, I. Routa, V. Sariola and Q. Zhou, J. Micromech. Microeng., 2011, 21, 095024.

63 T. Fukushima, T. Konno, E. Iwata, R. Kobayashi, T. Kojima, M. Murugesan, J. C. Bea, K. W. Lee, T. Tanaka and M. Koyanagi, Micromachines, 2011, 2, 49-68.

64 G. Arutinov, E. C. P. Smits, M. Mastrangeli, G. van Heck, J. van den Brand, H. F. M. Schoo and A. Dietzel, J. Micromech. Microeng., 2012, 22, 115022.

65 T. Fukushima, E. Iwata, Y. Ohara, A. Noriki, K. Inamura, K.-W. L. K.-W. Lee, J. B. J. Bea, T. Tanaka and M. Koyanagi, Electron Devices Meeting (IEDM), 2009 IEEE International, 2009, pp. 349-352, DOI: 10.1109/IEDM.2009.5424353.

66 J. Berthier, K. A. Brakke, S. Mermoz, C. Frétigny and L. Di Cioccio, Sens. Actuators, A, 2015, 234, 180-187.

67 K. L. Scott, R. T. Howe and C. J. Radke, 12th International Conference on Solid-State Sensors, Actuators and Microsystems (TRANSDUCERS '03), 2003, vol. 2, pp. 1319-1322.

68 K. F. Böhringer, U. Srinivasan and R. T. Howe, Micro Electro Mechanical Systems, 2001. MEMS 2001. The 14th IEEE International Conference on, 2001, pp. 369-374, DOI: 10.1109/MEMSYS.2001.906555.

69 X. Xiaorong, L. Sheng-Hsiung and K. F. Böhringer, Robotics and Automation, 2004. Proceedings. ICRA '04. 2004 IEEE International Conference on, 2004, vol. 2, pp. 1141-1148.

70 S. Arscott, E. Peytavit, D. Vu, A. C. H. Rowe and D. Paget, J. Micromech. Microeng., 2010, 20, 025023.

71 F. Rose, M. Hattori, D. Kobayashi, H. Toshiyoshi, H. Fujita and H. Kawakatsu, J. Micromech. Microeng., 2006, 16, 2077.

72 K. Sato, K. Ito, S. Hata and A. Shimokohbe, Precis. Eng., 2003, 27, 42-50.

73 J. L. M. Taprogge, F. Beyeler, A. Steinecker and B. J. Nelson, IEEE Trans. Compon., Packag., Manuf. Technol., 2013, 3, 1420-1429.

74 I. Routa, B. Chang, A. Shah and Q. Zhou, J. Microelectromech. Syst., 2014, 23, 819-828.

75 G. Arutinov, M. Mastrangeli, G. van Heck, P. Lambert, J. M. J. den Toonder, A. Dietzel and E. C. P. Smits, IEEE Transactions on Robotics, 2015, 31, 1033-1043.

76 K. S. Park, X. Xiong, R. Baskaran and K. F. Böhringer, J. Micromech. Microeng., 2011, 21, 025002.

77 K. S. Park, J. H. Hoo, R. Baskaran and K. F. Böhringer, J. Microelectromech. Syst., 2013, 22, 13-15.

78 R. J. Knuesel, S. Park, W. Zheng and H. O. Jacobs, J. Microelectromech. Syst., 2012, 21, 85-99.
79 V. Liimatainen, M. Kharboutly, D. Rostoucher, M. Gauthier and Q. Zhou, IEEE International Conference on Robotics and Automation, 2013, pp. 1403-1408, DOI: 10.1109/ ICRA.2013.6630754.

80 E. E. Kuran and M. Tichem, IEEE Trans. Autom. Sci. Eng., 2013, 10, 536-544.

81 J. B. Valsamis, M. Mastrangeli and P. Lambert, J. Mec. Theor. Appl., 2013, 38, 47-57.

82 N. van Veen, J. Electron. Packag., 1999, 121, 116-1212.

83 H. Lu and C. Bailey, IEEE Trans. Adv. Packag., 2005, 28, 475-480.

84 V. Dubey, J. Derakhshandeh, E. Beyne, J. P. Celis and I. D. Wolf, IEEE Trans. Compon., Packag., Manuf. Technol., 2016, 6, 946-953.

85 V. Sariola, PhD thesis, Aalto University, 2012.

86 J. M. Kim, Y. E. Shin and K. Fujimoto, Microelectron. Reliab., 2004, 44, 983-992.

87 J.-M. Kim, K. Yasuda and K. Fujimoto, J. Electron. Packag., 2005, 127, 18-24.

88 Delo-Dot PN2, https://www.delo-adhesives.com/en/dispensingvalve/delo-dot-pn2-micro-dispensing-valve/.

89 Microdrop Dispenser Heads MD-K-130, MD-K-140, http:// www.microdrop.com/Microdrop_Dispenser_Heads.html.

90 Medispec Sub-Microliter Piezoelectric Dispensers, http:// www.medispec.in/Dispensers.html.

91 Nordson EFD Fluidic Dispensing Systems, http://www. nordson.com/en/divisions/efd/products/fluid-dispensingsystems.

92 J. Fang and K. F. Böhringer, J. Microelectromech. Syst., 2006, 15, 531-540.

93 A. A. Darhuber, S. M. Troian, J. M. Davis, S. M. Miller and S. Wagner, J. Appl. Phys., 2000, 88, 5119.

94 R. W. Wood and A. L. Loomis, Philos. Mag., 1927, 4, 417-436.

95 E. K. Sackmann, L. Majlof, A. Hahn-Windgassen, B. Eaton, T. Bandzava, J. Daulton, A. Vandenbroucke, M. Mock, R. G. Stearns, S. Hinkson and S. S. Datwani, J. Lab. Autom., 2016, 21, 166-177.

96 B. Chang, A. Shah, Q. Zhou, R. H. A. Ras and K. Hjort, Sci. Rep., 2015, 5, 14966.

97 B. Chang, Q. Zhou, R. H. Ras, A. Shah, Z. Wu and K. Hjort, Appl. Phys. Lett., 2016, 108, 154102.

98 M. Kong, S. Jeon, H. Au, C. Hwang and Y.-C. Lee, IEEE Trans. Compon., Packag., Manuf. Technol., 2011, 1, 1523-1532.

99 W. Pittroff, J. Barnikow, A. Klein, P. Kurpas, U. Merkel, K. Vogel, J. Würfl and J. Kuhmann, Proc. 47th Electronic Components and Technology Conf., 1997, pp. 1235-1241, DOI: 10.1109/ECTC.1997.606333.

100 M. Wale and C. Edge, IEEE Trans. Compon., Hybrids, Manuf. Technol., 1990, 13, 780-786.

101 R. Zhao, Y. Zhang, R. W. Johnson and D. K. Harris, Proc. 51st Electronic Components and Technology Conf., 2001, pp. 699-703, DOI: 10.1109/ECTC.2001.927809.

102 J. R. Ellis and G. Y. Masada, IEEE Trans. Compon., Hybrids, Manuf. Technol., 1990, 13, 545-552.

103 R. J. K. Wassink and J. a. H. V. Gerven, Soldering Surf. Mount Technol., 1989, 1, 5-10. 
104 Q. Tan and Y. Lee, Electronic Components and Technology Conference, 1996. Proceedings., 46th, 1996, pp. 26-36.

105 T. H. Ju, W. Lin, Y. C. Lee, D. J. McKnight and K. M. Johnson, IEEE Photonics Technol. Lett., 1995, 7, 1010-1012.

106 M. F. Dautartas, A. M. Benzoni, S. L. Broutin, A. Coucoulas, D. T. Moser, Y. H. Wong and Y. M. Wong, IEEE Trans. Compon., Packag., Manuf. Technol., Part B, 1996, 19, 554-561.

107 M. F. Dautartas, G. E. Blonder, Y.-H. Wong and Y. C. Chen, IEEE Trans. Compon., Packag., Manuf. Technol., Part B, 1995, 18, 552-557.

108 G. Lecarpentier, J. Mottet, J. Dumas and K. Cooper, 2000 Proceedings. 50th Electronic Components and Technology Conference, 2000, DOI: 10.1109/ECTC.2000.853099.

109 J. Sasaki, M. Ito, T. Tamanuki, H. Hatakeyama, S. Kitamura, T. Shimoda and T. Kato, IEEE Trans. Adv. Packag., 2001, 24, 569-575.

110 T. Hayashi, IEEE Trans. Compon., Hybrids, Manuf. Technol., 1992, 15, 225-230.

111 C. J. Morris and B. A. Parviz, J. Micromech. Microeng., 2008, 18, 015022.

112 E. Saeedi, S. Abbasi, K. F. Böhringer and B. A. Parviz, FDMP: Fluid Dynamics \& Materials Processing, 2006, 2, 221-246.

113 T. Fukushima, E. Iwata, T. Konno, J.-C. Bea, K.-W. Lee, T. Tanaka and M. Koyanagi, Appl. Phys. Lett., 2010, 96, 154105.

114 Y. A. Chapuis, A. Debray, L. Jalabert and H. Fujita, J. Micromech. Microeng., 2009, 19, 1-9.

115 P. Soussan, W. Zhang, M. Pantouvaki, T. Delande and S. Armini, Proceedings of 2012 3rd IEEE International Workshop on Low Temperature Bonding for 3D Integration, LTB-3D 2012, 2012, vol. 33, p. 153.

116 T. Fukushima, E. Iwata, Y. Ohara, M. Murugesan, J. Bea, K. Lee, T. Tanaka and M. Koyanagi, IEEE Trans. Electron Devices, 2012, 59, 2956-2963.

117 Q. Zhou and B. Chang, Proc. IEEE/RSJ Int. Conf. Intelligent Robots \& Systems, 2006, pp. 5883-5888, DOI: 10.1109/ IROS.2006.282466.

118 L. Gao and T. J. McCarthy, Langmuir, 2007, 23, 10445-10447.

119 S. Zhang, N. Sun, X. He, X. Lu and X. Zhang, J. Phys. Chem. Ref. Data, 2006, 35, 1475-1517.

120 K. Sato, K. Lee, M. Nishimura and K. Okutsu, Int. J. Precis. Eng. Manuf., 2007, 8, 75-79.

121 R. J. Good and L. a. Girifalco, J. Phys. Chem., 1960, 24, 561-565.

122 K.-S. Moon, J. Wu and C. P. Wong, Proc. Int. Symp. on Advanced Packaging Materials: Processes, Properties and Interfaces, 2001, pp. 341-346.

123 C. del Corral, Q. Zhou, A. Albut, B. Chang, S. Franssila, S. Tuomikoski and H. N. Koivo, Proc. 2nd VDE World Microtechnology Congr., 2003, pp. 293-298.

124 T. Fukushima, E. Iwata, Y. Ohara, M. Murugesan, J. Bea, K. Lee, T. Tanaka and M. Koyanagi, IEEE Trans. Compon., Packag., Manuf. Technol., 2011, 1, 1873-1884.

125 R. Guerre, U. Drechsler, D. Jubin and M. Despont, J. Microelectromech. Syst., 2008, 17, 157-165.

126 R. Guerre, U. Drechsler, D. Bhattacharyya, P. Rantakari, R. Stutz, R. V. Wright, Z. D. Milosavljevic, T. Vaha-Heikkila,
P. B. Kirby and M. Despont, J. Microelectromech. Syst., 2010, 19, 548-560.

127 S. C. Park, J. Fang, S. Biswas, M. Mozafari, T. Stauden and H. O. Jacobs, J. Microelectromech. Syst., 2015, 24, 1928-1937.

128 J. S. Smith, International Electron Devices Meeting, 2000, pp. 201-204, DOI: 10.1109/IEDM.2000.904292.

129 H.-J. J. Yeh and J. S. Smith, IEEE Photonics Technol. Lett., 1994, 6, 706-708.

130 P. Lambert and A. Delchambre, Assembly Automation, 2005, 25, 275-283.

131 V. Sariola, V. Liimatainen, T. Tolonen, R. Udd and Q. Zhou, 2011 IEEE International Conference on Robotics and Automation, 2011, pp. 4098-4103, DOI: 10.1109/ICRA.2011.5979980.

132 S. Biswas, M. Mozafari, T. Stauden and H. Jacobs, Micromachines, 2016, 7, 54.

133 FC300R High Precision/Flip Chip Bonder, Smart Equipment Technology (SET), http://www.set-sas.fr/en/cat422407FC300.html?Cookie=set.

134 N. Karlitskaya, PhD thesis, University of Twente, 2011.

135 V. Marinov, O. Swenson, R. Miller, F. Sarwar, Y. Atanasov, M. Semler and S. Datta, IEEE Trans. Compon., Packag., Manuf. Technol., 2012, 2, 569-577.

136 V. R. Marinov, O. Swenson, Y. Atanasov and N. Schneck, Microelectron. Eng., 2013, 101, 23-30.

137 S. A. Stauth and B. A. Parviz, Proc. Natl. Acad. Sci. U. S. A., 2006, 103, 13922-13927.

138 Z. Wei, C. Jaehoon and H. O. Jacobs, J. Microelectromech. Syst., 2006, 15, 864-870.

139 J. P. Colonna, R. Segaud, F. Marion, M. Volpert, A. Garnier, L. D. Cioccio, Y. Beillard, S. Mermoz, F. D. Créchy, C. Laviron and S. Chéramy, 2013 IEEE 63rd Electronic Components and Technology Conference, 2013, pp. 872-878.

140 L. Sanchez, L. Bally, B. Montmayeul, F. Fournel, J. Dafonseca, E. Augendre, L. D. Cioccio, V. Carron, T. Signamarcheix, R. Taibi, S. Mermoz and G. Lecarpentier, 2012 IEEE 62nd Electronic Components and Technology Conference, 2012, pp. 1960-1964.

141 S. Mermoz, L. Sanchez, L. D. Cioccio, J. Berthier, E. Deloffre, P. Coudrain and C. Fretigny, Electronics Packaging Technology Conference (EPTC 2013), 2013 IEEE 15th, 2013, pp. 162-167.

142 EU FP7 Project FAB2ASM, http:/www.fab2asm.eu/.

143 J. L. M. Taprogge, PhD thesis, Eidgenössische Technische Hochschule ETH Zürich, Nr. 21030, 2013.

144 V. Sariola, Q. Zhou and H. N. Koivo, IEEE International Conference on Robotics and Automation, 2009, pp. 2605-2610.

$145 \mathrm{~J}$. Riseman, Beam-lead integrated circuit structure and method for making the same including automatic registration of beam-leads with corresponding dielectric substrate leads, US Pat., 4032058, IBM Corporation, Armonk, NY, 1977.

146 J. N. Elliott, Apparatus and method for aligning surface mountable electronic components on printed circuit board pads, US Pat., 4831724, Western Digital Corporation, Irvine, CA, 1989.

147 D. J. Pedder, A. D. Parsons and R. A. C. Bache, Manufacture of a hybrid electronic or optical device, Patent Application, WO/1987/001509, Plessey Overseas Limited, 1987. 
148 S. Tamaoki, Packaging of integrated circuit chip, Jpn. Pat., JPH0243748, Ricoh Co Ltd, 1990.

149 A. D. Hertz, D. A. Tribbey and K. R. Thompson, Self-aligning electrical contact array, Patent Application, WO/1994/ 000969 , Motorola, INC., 1994.

150 B. A. Parviz and S. Stauth, Fluidic self-assembly for system integration, Patent Application, WO/2007/150066, University of Washington, 2007.

151 P. D. Brewer, A. T. Hunter and L. M. Deckard, Method for assembly of complementary-shaped receptacle site and device microstructures, US Pat., 7018575, HRL Laboratories, LLC, Malibu, CA, US, 2006.

152 D. H. Gracias, J. Tien and G. M. Whitesides, Self-assembled electrical networks, US Pat., 7007370, President and Fellows of Harvard College, Cambridge, MA, US, 2006.

153 G. S. W. Craig, E. J. Snyder and J. K.-J. Tu, Methods and apparatuses relating to block configurations and fluidic selfassembly processes, US Pat., 6919225, Alien Technology Corporation, Morgan Hill, CA, US, 2005.

154 G. S. W. Craig, M. Chan X, C. V. Sutu, O. R. Alvarado, H. Pham and M. A. Hadley, Apparatus for forming an electronic assembly, US Pat., 7080444, Alien Technology Corporation, Morgan Hill, CA, US, 2006.

155 G. S. W. Craig, A. A. Tootoonchi, S. Herrmann, G. Gengel and R. Eisenhardt, Strap assembly comprising functional block deposited therein and method of making same, US Pat., 7452748, Alien Technology Corporation, Morgan Hill, CA, US, 2008.

156 T. L. Credelle, O. Alvarado, G. S. W. Craig, M. A. Hadley and K. D. Schatz, Method and apparatus for transferring blocks, US Pat., 6731353, Alien Technology Corporation, Morgan Hill, CA, USA, 2004.

157 G. W. Gengel, Integrated circuit packages assembled utilizing fluidic self-assembly, US Pat., 6566744, Alien Technology Corporation, Morgan Hill, CA, USA, 2003.

158 J. J. Jacobsen and R. G. Stewart, Apparatuses and methods for forming electronic assemblies, US Pat., 7417306, Alien Technology Corporation, Morgan Hill, CA, US, 2008.

159 K. D. Schatz, Method and apparatus for self-assembly of functional blocks on a substrate facilitated by electrode pairs, US Pat., 7321159, Alien Technology Corporation, Morgan Hill, CA, US, 2008.

160 J. S. Smith, M. A. Hadley, G. S. W. Craig and P. F. Nealey, Methods and apparatuses for assembling elements onto a substrate, US Pat., 7141176, Alien Technology Corporation, Morgan Hill, CA, US, 2006.
161 J. S. Smith, M. A. Hadley, G. S. W. Craig and F. Lowe, Methods and apparatuses for fluidic self assembly, US Pat., 7531218, Alien Technology Corporation, Morgan Hill, CA, US, 2009.

162 T. K. Ricks and R. Sharma, Method for assembling microcomponents to binding sites, US Pat., 7251882, Eastman Kodak Company, Rochester, NY, US, 2007.

163 D. Gracias, Fabricating stacked chips using fluidic templatedassembly, US Pat., 7375425, Intel Corporation, Santa Clara, CA, US, 2008.

164 D. K. Fork, T. Hantschel and M. L. Chabinyc, System including self-assembled interconnections, US Pat., 7525194, Palo Alto Research Center Incorporated, Palo Alto, CA, US, 2009.

165 J. S. Smith and H.-J. J. Yeh, Method for fabricating selfassembling microstructures, US Pat., 5545291, The Regents of the University of California, Oakland, CA, 1996.

166 J. S. Smith, Fluidic self-assembly of active antenna, US Pat., 6611237, The Regents of the University of California, Oakland, CA, 2003.

$167 \mathrm{H}$. Torii, Method for mounting anisotropically-shaped members, US Pat., 7528004, Panasonic Corporation, Osaka, JP, 2009.

168 S. Karashima, T. Kitae and S. Nakatani, Flip chip mounting method and bump forming method, US Pat., 7531387, Panasonic Corporation, Osaka, JP, 2009.

169 M. D. Rostoker and N. F. Pasch, Shaped, self-aligning microbump structures, US Pat., 5558271, LSI Logic Corporation, Milpitas, CA, 1996.

170 M. Koyanagi, Method and apparatus for manufacturing integrated circuit device using self organizing function, Patent Application, WO/2006/077739, Koyanagi, Mitsumasa, 2006.

171 M. Koyanagi, T. Fukushima and M. Sugiyama, Method and apparatus for manufacturing three-dimensional integrated circuit, US Pat., 20120021563, Tohoku University, Sendaishi, Miyagi, JP, Tokyo Electron Limited, Tokyo, JP, 2012.

172 L. Di Cioccio, F. Grossi, P. Gueguen and L. Vandroux, Selfassembly of chips on a substrate, US Pat. Appl., 20110033976, Commissariat à l'energie atomique et aux energies alternatives, Paris, FR, 2011.

173 M. M. Mueller, H. Zabel and H.-P. Monser, Method and apparatus for manufacturing an electronic assembly, Patent Application, WO/2010/066366, Muehlbauer AG, Josef-Mühlbauer-Platz 1, Roding, 93426, DE, 2010.

174 B. Chang, Q. Zhou, Z. Wu, Z. Liu, R. Ras and K. Hjort, Micromachines, 2016, 7, 41. 